\title{
The Hessian in Spin Foam Models
}

\author{
Wojciech Kamiński@i and Hanno Sahlmann
}

\begin{abstract}
We fill one of the remaining gaps in the asymptotic analysis of the vertex amplitudes of the Engle-Pereira-Rovelli-Livine (EPRL) spin foam models: We show that the Hessian is nondegenerate for the stationary points that corresponds to geometric nondegenerate 4 simplices. Our analysis covers the case when all faces are spacelike.
\end{abstract}

\section{Introduction}

One of the central results of the research on spin foam models (defined in $[1,2]$ and extended in [3]) is the asymptotic analysis of the vertex amplitude accomplished in [4-6] for the Euclidean case and in [7-9] for the Lorentzian case). The graviton propagator [10-12], the relation to Regge calculus and various semiclassical limits $[13,14]$ are all based on this result. Let us mention that exactly the asymptotic analysis [15] of the vertex of the Barrett-Crane model [16] led to the discovery of nongeometric sectors [17] and in consequence to the invention of the EPRL model. However, it is important to keep in mind that the analysis of the vertex amplitude does not capture all properties of the model - as seen by so-called flatness problem $[18,19]$ that is not visible in the asymptotics of a single vertex.

The proof of the asymptotic formula for various spin foam models is not completely water-tight because of a few issues. First of all, the proof is based on stationary phase method and typically integration is done over noncompact domains. It is not clear if there are any contributions from infinity or from boundary of the domain of integration. In the Hnybida-Conrady extension [3] it is even not known if the amplitude is finite at all. Secondly, the contribution from a stationary point depends on whether the point is nondegenerate (i.e. the Hessian at that point has no zero eigenvectors, after gauge fixing) or not. These issues were summarized in our previous paper [8].

The current paper is devoted to the problem of whether or not the Hessian is nondegenerate for a given stationary point. The only analytic result in this direction that we know about for $4 d$ models is the result [20] for the 
Barrett-Crane model [16]. For the Euclidean EPRL model, it was checked for specific examples that the Hessian is nondegenerate ${ }^{1}$ so its determinant is nonzero for generic boundary data. However, the example of the BarrettCrane model can serve as a warning, as in this case the Hessian is degenerate for configurations where the map from lengths to areas of the 4-simplex is not locally invertible. The Lorentzian models are more complicated. The number of integration variables makes the determination of the determinant of the Hessian an almost intractable task.

In this paper we will show that for the EPRL models in both, Euclidean and Lorentzian signature (we consider also Hnybida-Conrady extension), with spacelike faces, the Hessian is nondegenerate for every stationary point that corresponds to a nondegenerate 4-simplex (of either Lorentzian or other signature).

We will first consider the Euclidean EPRL model with Barbero-Immirzi parameter $\gamma<1$, as it can be treated in a considerably simpler way. The crucial observation for our analysis of this case is the specific behaviour of the Hessian for actions satisfying a certain reality condition: If $e^{i S}$ denotes the integrand of the amplitude, then the imaginary part of the action is nonnegative,

$$
\Im S \geq 0 \text {. }
$$

In order to extend our result to the case of Lorentzian models, we introduce a reduced action that is more closely related to the action of the Euclidean model. The reduced action is defined in such a way that non-degeneracy of its Hessian is equivalent to the non-degeneracy of that of the full action. We then reexpress the analysis of the Euclidean amplitude in symplectic geometric terms. The geometric theory of such actions is based on positive Lagrangians that were introduced by [21]. This makes it applicable to the Lorentzian case as well.

The main reference for our notation is [8]. There are a few departures from that notation, for which we refer the reader to "Appendix A".

\section{Euclidean EPRL Model with $\gamma<1$}

In the following, our terminology and, in particular what is real and what is imaginary is based on the convention that the integrand of the integral we are approximating is $e^{i S}$, and we will cal $S$ the action. We note that this is different from the convention of [7].

For a symmetric (or Hermitian) form $H$ we will use the notation

$$
H v=H(\cdot, v) \text {. }
$$

We will say that the vector $v$ annihilates $H$ if

$$
H v=0 .
$$

For a real symmetric form $I$ we write $I \geq 0$ if for any real vector $w$

$$
I(w, w) \geq 0 .
$$

\footnotetext{
${ }^{1}$ Frank Hellmann, private communication.
} 
This is equivalent to the condition that for any complex vector $v$

$$
I(\bar{v}, v) \geq 0 .
$$

Lemma 1. Assume that the symmetric complex form $H$ can be decomposed as $H=R+i I$ where $R$ and $I$ are real and $I \geq 0$. Then the following conditions for a vector $v$ are equivalent:

1. $v$ annihilates $H$

$$
H v=0 .
$$

2. The following is true for the real and imaginary part of the vector $v$ $(v=\Re v+i \Im v)$ :

$$
R \Re v=R \Im v=I \Re v=I \Im v=0 .
$$

Proof. Let us write $v=v_{r}+i v_{a}$ where $v_{r}$ and $v_{a}$ are real.

We have from the linearity of the forms

$$
0=H v=\left(R v_{r}-I v_{a}\right)+i\left(R v_{a}+I v_{r}\right),
$$

thus $R v_{r}=I v_{a}$ and $R v_{a}=-I v_{r}$. Moreover, from the symmetry of $R$

$$
I\left(v_{a}, v_{a}\right)=R\left(v_{a}, v_{r}\right)=R\left(v_{r}, v_{a}\right)=-I\left(v_{r}, v_{r}\right) .
$$

As $I \geq 0$ we see that $I\left(v_{a}, v_{a}\right)=0$ and $I\left(v_{r}, v_{r}\right)=0$, thus

$$
I v_{r}=I v_{a}=0
$$

and also $R v_{r}=R v_{a}=0$.

Lemma 2. Suppose that the symmetric real form $I=\sum_{\alpha} I_{\alpha}$ and $I_{\alpha} \geq 0$. Then

$$
I v=0 \Longleftrightarrow \forall_{\alpha} I_{\alpha} v=0 \text {. }
$$

Proof. We have $I(\bar{v}, v)=0$ thus $\sum_{\alpha} I_{\alpha}(\bar{v}, v)=0$. All terms are positive, thus each of them needs to be zero, but due to positivity this implies that $I_{\alpha} v=0$.

\subsection{Hessian in Euclidean EPRL}

The manifold of integration is $\prod_{i=1}^{4} \operatorname{Spin}(4)$ and thus the vectors of the tangent space can be described by

$$
v:\{1, \ldots, 5\} \rightarrow \mathbb{R}^{3} \oplus \mathbb{R}^{3}, \quad v(5)=0 .
$$

We will denote self-dual (antiself-dual) part by $v^{ \pm}$. $[5]^{2}$

The tensor of second derivatives of the action (the Hessian) is given by

$$
H\left(v, v^{\prime}\right)=H^{+}\left(v^{+}, v^{+}\right)+H^{-}\left(v^{-}, v^{\prime-}\right) .
$$

Let us consider the self-dual part (the antiself-dual is analogous). We can write $H^{+}$as

$$
H=R+i\left(\sum_{1 \leq a<b \leq 5} I_{a b}\right)
$$

\footnotetext{
${ }^{2}$ Published version.
} 
where $I_{a b}$ are given by

$$
I_{a b}\left(v^{+}, v^{+}\right)=I_{a b}^{\prime}\left(v^{+}(a)-v^{+}(b), v^{+}(a)-v^{+}(b)\right)
$$

in terms of symmetric real forms $I_{a b}^{\prime}: \mathbb{R}^{3} \times \mathbb{R}^{3} \rightarrow \mathbb{R}$

$$
I_{a b}^{\prime}(w, w)=\frac{j_{a b}^{+}}{2}\left(|w|^{2}-\left(w \cdot n_{a b}^{+}\right)^{2}\right) .
$$

This form is $\frac{j_{a b}^{+}}{2}$ times the expectation value of the projector onto the space perpendicular to $n_{a b}^{+}$, so it is nonnegative $\left(\frac{j_{a b}^{+}}{2} \geq 0\right)$, thus also $I_{a b} \geq 0$.

The real form $R$ is given by

$$
R\left(v, v^{\prime}\right)=\sum_{a, b \in\{1, \ldots 5\}} \frac{j_{a b}^{+}}{2} n_{a b} \cdot v^{+}(a) \times v^{\prime+}(b),
$$

where we use the convention that $n_{a b}=-n_{b a}$.

Lemma 3. If $\operatorname{det} H=0$ then there exist $a \neq b \in\{1, \ldots 4\}$ such that $n_{a 5}, n_{a b}$, $n_{b 5}$ are linearly dependent.

Proof. If $\operatorname{det} H=0$ then there exists a nonzero vector $v^{\prime}$ such that $H v^{\prime}=$ 0 thus Lemma 1 assures that there exists a nonzero real vector $v$ that is annihilated by $\Im H$. It needs to be annihilated by every $I_{a b}$ due to Lemma 2 . The conditions

$$
I_{5 a} v=0, \quad I_{5 b} v=0
$$

give

$$
v(a)=\lambda_{b} n_{a 5}, \quad v(b)=\lambda_{a} n_{b 5},
$$

where $\lambda_{k} \in \mathbb{R}$. The condition $I_{a b} v=0$ gives

$$
v(a)-v(b)=\lambda_{5} n_{a b},
$$

thus

$$
\lambda_{b} n_{a 5}-\lambda_{a} n_{b 5}-\lambda_{5} n_{a b}=0 .
$$

Either $v(a)=v(b)=0$ or $n_{a b}, n_{a 5}, n_{b 5}$ are linearly dependent.

As this is true for all $a, b$ we have either $v=0$ (contradiction) or there exist $a, b$ fulfilling the statement of the lemma.

Theorem 1. The Hessian for the Euclidean EPRL model with $\gamma<1$ is nondegenerate for any stationary point that corresponds to a nondegenerate 4simplex.

Proof. If $n_{a b}, n_{a 5}, n_{b 5}$ are linearly dependent then the matrix $\tilde{G}_{a b 5}$ defined in equation (301) from [8] is degenerate, and lemma 28 from [8] (in its version for Euclidean signature) tells us that there exists at most one stationary point (a single vector geometry or a degenerate 4 -simplex).

For the case of Euclidean EPRL just considered the integration is over the compact manifold; thus, the nondegeneracy of the Hessian was the only missing part of the asymptotic analysis. We will not consider Euclidean case with $\gamma>1$ because it can be treated in an analogous way to the Lorentzian case. We will now describe the Lorentzian case in detail. 


\section{Extension to the Lorentzian EPRL Amplitude}

In the case of the Lorentzian EPRL amplitude, integration is over many more variables and the Hessian is more complicated. The action is a sum ${ }^{3}$

$$
\tilde{S}\left(\left\{g_{i}\right\},\left\{\mathbb{Z}_{i j}\right\}\right)=\sum_{1 \leq i<j \leq 5} \tilde{S}_{i j}\left(g_{i}, g_{j}, \mathbb{Z}_{i j}, \mathbb{Z}_{j i}\right),
$$

where

$$
\tilde{S}_{i j}\left(g_{i}, g_{j}, \mathbb{Z}_{i j}, \mathbb{Z}_{j i}\right)=S_{i j}^{n_{i j}}\left(g_{i}^{-1} \mathbb{Z}_{i j}\right)+S_{i j}^{\beta}\left(\mathbb{Z}_{i j}, \mathbb{Z}_{j i}\right)+S_{j i}^{n_{j i}}\left(g_{j}^{-1} \mathbb{Z}_{j i}\right) .
$$

Actions as well as measure factors are at least locally analytic. If we denote by $\left[\mathbb{Z}_{i j}\right]$ elements of $\mathbb{C P}$ (i.e., equivalence classes of spinors) then the stationary points are discrete and we are interested in one of them

$$
g_{i}^{0},\left[\mathbb{Z}_{i j}^{0}\right],
$$

where $g_{5}^{0}=1$. We will denote bivectors (see Sect. 4.1 and "Appendix A" for notation $^{4}$ )

$$
B_{i j}^{0}=\delta_{\mathbb{Z}_{i j}} S_{i j}^{\beta},
$$

and we will write $B_{i j}^{0^{\prime}}=g_{i}^{-1} B_{i j}^{0}$ for a bivector in the node frame. We will call it the fundamental stationary point.

\subsection{Reduced Action}

The variables $\left\{g_{i}\right\}$ appear in many places, but for fixed $i j$ the variables $\mathbb{Z}_{i j}$ and $\mathbb{Z}_{j i} \in \mathbb{C P}$ are only found in the action $\tilde{S}_{i j}$. Let us denote the form of second derivatives with respect to the $\mathbb{C P}$ variables by $H_{\mathbb{Z Z Z}}$. It is block diagonal, with blocks corresponding to $\left\{\mathbb{Z}_{i j}, \mathbb{Z}_{j i}\right\}$. We will show later that this form is nondegenerate (in the neighbourhood of the fundamental stationary point).

Let us (locally) analytically extend the action in the $\mathbb{Z}$ variables to the complexification $\mathbb{C P}^{\mathbb{C}}, 5$

$$
\tilde{S}\left(\left\{g_{i}\right\},\left\{\mathbb{Z}_{i j}^{\mathbb{C}}\right\}\right) .
$$

Let us notice that $\tilde{S}$ depends only on the $\mathbb{C P}$ variables $\left[\mathbb{Z}_{i j}^{\mathbb{C}}\right]$ (equivalence classes of spinors). As the Hessian $H_{\mathbb{Z Z Z}}$ is nondegenerate at the fundamental stationary point we can (in the neighbourhood of $g_{i}^{0}$ ) find a unique (in the neighbourhood of $\left.\left[\mathbb{Z}_{i j}\right]^{0}\right)$ solution

$$
\left[\mathbb{Z}_{i j}^{\mathbb{C}}\right]: \forall i j \frac{\partial \tilde{S}}{\partial\left[\mathbb{Z}_{i j}^{\mathbb{C}}\right]}=0 .
$$

Here $\frac{\partial \tilde{S}}{\partial\left[\mathbb{Z}_{i j}^{\mathbb{C}}\right]}$ is a holomorphic derivative as the antiholomorphic one gives $\frac{\partial \tilde{S}}{\partial\left[\mathbb{Z}_{i j}^{\mathbb{C}}\right]}=$ 0 everywhere. Let us notice that due to the form of the action the solution has a specific dependence on $\left\{g_{i}\right\}$

\footnotetext{
${ }^{3}$ We will use the notation from [8]. A summary of notation and conventions is also in "Appendix A".

${ }^{4}$ It differs slightly from [8] due to other normalization constants in the scalar product and some sign factors.

${ }^{5}$ We regard $\mathrm{SL}(2, \mathbb{C})$ and $\mathbb{C P}$ as real manifolds. Complexification of a space $M$ that is already a complex manifold gives a space $M \times \bar{M}$.
} 


$$
\left[\mathbb{Z}_{i j}^{\mathbb{C}}\right]\left(g_{i}, g_{j}\right)
$$

Let us introduce a reduced action

$$
S^{\mathrm{red}}\left(\left\{g_{i}\right\}\right)=\sum_{i<j} S_{i j}^{\mathrm{red}}\left(g_{i}, g_{j}\right), S_{i j}^{\mathrm{red}}\left(g_{i}, g_{j}\right)=\tilde{S}_{i j}\left(g_{i}, g_{j},\left[\mathbb{Z}_{i j}^{\mathbb{C}}\right]\left(g_{i}, g_{j}\right),\left[\mathbb{Z}_{j i}^{\mathbb{C}}\right]\left(g_{j}, g_{i}\right)\right) \text {. }
$$

The point $g_{i}^{0}$ is a stationary point of this action and the Hessian at this point is $H^{\text {red }}=\sum H_{i j}^{\text {red }}$. Let us notice that $S_{i j}^{\text {red }}$ depends only on the group element $g_{i j}=g_{j}^{-1} g_{i}$

$$
S_{i j}^{\mathrm{red}}\left(g_{i}, g_{j}\right)=S_{i j}^{\prime}\left(g_{i j}\right) \text {. }
$$

We have the projection map on the complexified tangent space

$$
\Pi: T_{\left\{g_{i}^{0},\left[\mathbb{Z}_{i j}^{0}\right]\right\}}^{\mathbb{C}}\left(\prod_{i} \mathrm{SL}(2, \mathbb{C}) \times \prod_{i \neq j} \mathbb{C P}\right) \rightarrow T_{\left\{g_{i}^{0}\right\}}^{\mathbb{C}}\left(\prod_{i} \mathrm{SL}(2, \mathbb{C})\right) .
$$

We can also introduce a cross section

$$
\begin{aligned}
& \Xi: T_{\left\{g_{i}^{0}\right\}}^{\mathbb{C}}\left(\prod_{i} \mathrm{SL}(2, \mathbb{C})\right) \rightarrow T_{\left\{g_{i}^{0},\left[\mathbb{Z}_{i j}^{0}\right]\right\}}^{\mathbb{C}}\left(\prod_{i} \mathrm{SL}(2, \mathbb{C}) \times \prod_{i \neq j} \mathbb{C P}\right), \\
& \Xi(V)=V+\sum_{i j} V \mathbb{Z}_{i j}^{\mathbb{C}}\left(g_{i}, g_{j}\right) \frac{\partial}{\partial\left[\mathbb{Z}_{i j}^{\mathbb{C}}\right]}+V \overline{\mathbb{Z}_{i j}^{\mathbb{C}}}\left(g_{i}, g_{j}\right) \frac{\partial}{\partial\left[\overline{\left.\mathbb{Z}_{i j}^{\mathbb{C}}\right]}\right.} .
\end{aligned}
$$

We also use these maps restricted to fixed $i j$ sectors $\left(\Pi_{i j}\right.$ and $\left.\Xi_{i j}\right)$.

Lemma 4. The following holds:

$$
H_{i j}^{\mathrm{red}}\left(\Pi_{i j}\left(W_{i j}\right), V_{i j}\right)=H_{i j}\left(W_{i j}, \Xi_{i j}\left(V_{i j}\right)\right) .
$$

Also

$$
H^{\mathrm{red}}(\Pi(W), V)=H(W, \Xi(V)) .
$$

Proof. Due to the condition $(27)$ on $\mathbb{Z}^{\mathbb{C}}$ we have for $W_{i j} \in(T \operatorname{SL}(2, \mathbb{C}))^{2} \times$ $(T \mathbb{C P})^{2}$

$$
\begin{aligned}
& W_{i j}\left(\Im S_{i j}\left(g_{i}, g_{j}, \mathbb{Z}_{i j}^{\mathbb{C}}\left(g_{i}, g_{j}\right), \mathbb{Z}_{j i}^{\mathbb{C}}\left(g_{i}, g_{j}\right)\right)\right) \\
& =\left(\Pi_{i j}\left(W_{i j}\right) \Im S_{i j}\right)\left(g_{i}, g_{j}, \mathbb{Z}_{i j}^{\mathbb{C}}\left(g_{i}, g_{j}\right), \mathbb{z}_{j i}^{\mathbb{C}}\left(g_{i}, g_{j}\right)\right) .
\end{aligned}
$$

Let us notice that for $V_{i j} \in T \mathrm{SL}(2, \mathbb{C})^{2}$

$$
\begin{aligned}
& V_{i j}\left(\left(\Pi_{i j}\left(W_{i j}\right) \Im S_{i j}\right)\left(g_{i}, g_{j}, \mathbb{Z}_{i j}^{\mathbb{C}}\left(g_{i}, g_{j}\right), \mathbb{Z}_{j i}^{\mathbb{C}}\left(g_{i}, g_{j}\right)\right)\right) \\
& =V_{i j}\left(W_{i j} \Im S_{i j}\right)\left(g_{i}, g_{j}, \mathbb{Z}_{i j}^{\mathbb{C}}\left(g_{i}, g_{j}\right), \mathbb{Z}_{j i}^{\mathbb{C}}\left(g_{i}, g_{j}\right)\right) \\
& \quad=\left(\Xi_{i j}\left(V_{i j}\right) W_{i j} \Im S_{i j}\right)\left(g_{i}, g_{j}, \mathbb{Z}_{i j}^{\mathbb{C}}\left(g_{i}, g_{j}\right), \mathbb{Z}_{j i}^{\mathbb{C}}\left(g_{i}, g_{j}\right)\right) .
\end{aligned}
$$

Thus $H_{i j}^{\text {red }}\left(V_{i j}, \Pi_{i j}\left(W_{i j}\right)\right)=H_{i j}\left(\Xi_{i j}\left(V_{i j}\right), W_{i j}\right)$. Summing over $i j$ we get also the second equality.

Lemma 5. The Hessian is degenerate if and only if the reduced Hessian is. 
Proof. Let suppose that $H V=0$, then for any $W$

$$
0=H(\Xi(W), V)=H^{\text {red }}(W, \Pi(V)),
$$

thus $H^{\text {red }} \Pi(V)=0$. The other way around, if $H^{\text {red }} W=0$ then for any $V$

$$
H(\Xi(W), V)=H^{\mathrm{red}}(W, \Pi(V))=0,
$$

thus $H \Xi(W)=0$.

Definition 1. An extremal point of the action $S$ is a point on the real manifold where $\partial \Im S=0$ and the tensor of second derivatives of $\Im S$ is nonnegative definite.

If the action $S$ satisfies the reality condition (1) $(\Im S \geq 0)$ then points on the real manifold where $\Im S=0$ are extremal. The fundamental stationary point $\left\{g_{i}^{0},\left[\mathbb{Z}_{i j}\right]^{0}\right\}$ is extremal for the actions $\tilde{S}_{i j}$. The following is a consequence:

Lemma 6. The Hermitian form

$$
\Im H_{i j}^{\mathrm{red}}\left(\overline{V_{i j}}, V_{i j}\right)
$$

is nonnegative definite.

Proof. The maps $\Xi_{i j}$ and $\Pi_{i j}$ are compatible with complex conjugation thus

$$
\begin{aligned}
& \Im H_{i j}^{\mathrm{red}}\left(\overline{V_{i j}}, V_{i j}\right)=\Im H_{i j}^{\mathrm{red}}\left(\overline{V_{i j}}, \Pi_{i j} \Xi_{i j}\left(V_{i j}\right)\right)= \\
& \left.=\Im H_{i j}\left(\Xi_{i j}\left(\overline{V_{i j}}\right), \Xi_{i j}\left(V_{i j}\right)\right)=\Im H_{i j}\left(\overline{\Xi_{i j}\left(V_{i j}\right.}\right), \Xi_{i j}\left(V_{i j}\right)\right) \geq 0,
\end{aligned}
$$

because the imaginary part of the Hessian $H_{i j}$ is nonnegative definite.

Let us summarize:

Lemma 7. The point $\left\{g_{i}^{0}\right\}$ is an extremal point of $S_{i j}^{\mathrm{red}}$.

\section{Symplectic Geometry}

We will adapt the theory of positive Lagrangians introduced in [21]. Let $\Omega$ be the symplectic form on $T^{*} M$. It is the inverse to the Poisson bracket

$$
\Omega(v,\{D, \cdot\})=v(D), \quad v \in T\left(T^{*} M\right), D \in C^{\infty}\left(T^{*} M\right) .
$$

Let us consider an analytic function $S: M \rightarrow \mathbb{C}$ (maybe defined only on an open set $U$ ). The manifold

$$
\mathcal{L}_{S}=\{(x, p): \theta=d S(x)\} \subset T^{* \mathbb{C}} M
$$

is Lagrangian, that is it extends analytically to an analytic Lagrangian submanifold of $T^{*} M^{\mathbb{C}}$ in some neighbourhood of the real $T^{*} M$. Here we denoted by $\theta$ the tautological form $\theta=p_{\mu} d x^{\mu}$.

Over real points of $M$ the complex conjugation of the tangent space of the Lagrangian, $\overline{T^{\mathbb{C}} \mathcal{L}_{S}}$ is in itself the tangent space of the holomorphic Lagrangian

$$
\mathcal{L}_{\bar{S}}=\{(x, p): \theta=d \bar{S}(x)\} \subset T^{* \mathbb{C}} M .
$$

The tangent space of $\mathcal{L}_{S}$ can be identified by projection $\pi: T^{*} M \rightarrow M$ with the tangent space of $M$. We will denote this map by $\Pi_{S}: T^{\mathbb{C}} \mathcal{L} \rightarrow T^{\mathbb{C}} M$.

Now we will state and prove some important facts about extremal points: 
Lemma 8. The following holds for an extremal point $x_{0}$ of the action $S$

1. $p_{0}=d S\left(x_{0}\right)$ is real.

2. The Hermitian form on $T_{\left(x_{0}, p_{0}\right)}^{\mathbb{C}} \mathcal{L}$

$$
I\left(v, v^{\prime}\right)=-\frac{i}{2} \Omega_{\left(x_{0}, p_{0}\right)}\left(\bar{v}, v^{\prime}\right), \quad v, v^{\prime} \in T_{\left(x_{0}, p_{0}\right)}^{\mathbb{C}} \mathcal{L}
$$

is nonnegative definite and

$$
I\left(v, v^{\prime}\right)=\partial^{2} \Im S\left(\Pi_{S} \bar{v}, \Pi_{S} v^{\prime}\right) .
$$

3. Let $w \in T_{x_{0}} M^{\mathbb{C}}$ and we denote $v=\Pi_{S}^{-1} w$ then

$$
\left.I v=0 \text { (that is: }\left(\partial^{2} \Im S\right) w=0\right)
$$

is equivalent to

$$
v \in T_{\left(x_{0}, p_{0}\right)}^{\mathbb{C}} \mathcal{L}_{S} \cap \overline{T_{\left(x_{0}, p_{0}\right)}^{\mathbb{C}} \mathcal{L}_{S}}
$$

Proof. At an extremal point $p=d \Re S$ because derivatives of imaginary parts vanish. Let us use local coordinates $p_{\mu}, x^{\mu}$ on $T^{*} M$ then

$$
\left\{p_{\mu}-\frac{\partial \bar{S}}{\partial x^{\mu}}, p_{\nu}-\frac{\partial S}{\partial x^{\nu}}\right\}=2 i \frac{\partial^{2} \Im S}{\partial x^{\mu} \partial x^{\nu}} .
$$

Every vector tangent to $\mathcal{L}$ can be written as

$$
V=f^{\mu}\left\{p_{\mu}-\partial_{\mu} S, \cdot\right\}
$$

where $f^{\mu}$ are some complex constants. Thus at the point $\left(x_{0}, p_{0}\right)$

$$
-\frac{i}{2} \Omega(\bar{V}, V)=-\frac{i}{2} \overline{f^{\mu}} f^{\nu}\left\{p_{\mu}-\partial_{\mu} \bar{S}, p_{\nu}-\partial_{\nu} S\right\}=\overline{f^{\mu}} f^{\nu} \partial_{\mu} \partial_{\nu} \Im S .
$$

From tensoriality of the second derivative at a point where $\partial \Im S=0$, we get

$$
-\frac{i}{2} \Omega(\bar{V}, V)=\partial^{2} \Im S\left(\overline{\Pi_{S} V}, \Pi_{S} V\right),
$$

thus it is nonnegative definite. Let $V \in T^{\mathbb{C}} \mathcal{L}_{S}$ be such that $I V=0$ then

$$
\Omega(V, W)=0
$$

for all $W \in \overline{T^{\mathbb{C}} \mathcal{L}_{S}}=T^{\mathbb{C}} \mathcal{L}_{\bar{S}}$ thus $V \in T^{\mathbb{C}} \mathcal{L}_{\bar{S}}$.

We need some definition.

Definition 2. We will say that the Lagrangian $\mathcal{L}$ at the real point $\left(x_{0}, p_{0}\right)$ is positive if

$$
I_{\left(x_{0}, p_{0}\right)}\left(v, v^{\prime}\right)=-\frac{i}{2} \Omega\left(\bar{v}, v^{\prime}\right)
$$

is nonnegative definite. We will say that it is strictly positive if additionally $I_{\left(x_{0}, p_{0}\right)}$ is nondegenerate (has no zero vectors).

A Lagrangian is strictly positive if and only if

$$
T_{\left(x_{0}, p_{0}\right)}^{\mathbb{C}} \mathcal{L} \cap \overline{T_{\left(x_{0}, p_{0}\right)}^{\mathbb{C}} \mathcal{L}}=\{0\},
$$

that is, the only real vector in $T_{\left(x_{0}, p_{0}\right)}^{\mathbb{C}} \mathcal{L}$ is the trivial vector. 
Let $f$ be an analytic function on $T^{*} M$ (it extends locally to $T^{*} M^{\mathbb{C}}$ ) that vanishes on $\mathcal{L}_{S}$. The complex vector field

$$
\{f, \cdot\}
$$

is tangent to $\mathcal{L}_{S}$. If at the real point the Lagrangian is positive, then

$$
-i\{\bar{f}, f\}=-i \Omega(\{\bar{f}, \cdot\},\{f, \cdot\}) \geq 0 .
$$

\subsection{Symplectic Theory of $T^{*} \mathrm{SL}(2, \mathbb{C})$}

The left invariant vector field $\mathbb{L}(L)$ of the Lie algebra element $L$ corresponds to the first order jets of $g \rightarrow g e^{t L}$. The right invariant vector field $\mathbb{R}(L)$ of the same Lie algebra element will be $g \rightarrow e^{-t L} g$ (the sign is necessary for proper commutation relations).

With every point of the cotangent bundle $T^{*} \mathrm{SL}(2, \mathbb{C})$, we can associate a left and a right coalgebra element $p^{L}$ and $p^{R}$ given by the formula

$$
\forall L \in \operatorname{so}(1,3) \quad p^{L}(L)=\theta(\mathbb{L}(L)), \quad p^{R}(L)=\theta(\mathbb{R}(L)) .
$$

Let us notice that at the base point $g$

$$
p^{R}=-g^{-1} \cdot p^{L},
$$

where $g$ acts on the coalgebra by the co-adjoint action (if we identify the coalgebra with bivectors using the scalar product, then the coadjoint action is the same as the adjoint action, see "Appendix A"). For any Lie algebra element $L$,

$$
p^{L}(L), p^{R}(L)
$$

are functions on $T^{*} \mathrm{SL}(2, \mathbb{C})$. We have

$$
\begin{gathered}
\left\{p^{L}(L), p^{L}\left(L^{\prime}\right)\right\}=-p^{L}\left(\left[L, L^{\prime}\right]\right),\left\{p^{R}(L), p^{R}\left(L^{\prime}\right)\right\}=-p^{R}\left(\left[L, L^{\prime}\right]\right), \\
\left\{p^{L}(L), p^{R}\left(L^{\prime}\right)\right\}=0,
\end{gathered}
$$

and also

$$
\left\{f(g), p^{L}(L)\right\}=\mathbb{L}(L) f,\left\{f(g), p^{R}(L)\right\}=\mathbb{R}(L) f .
$$

Let us denote by $\delta^{L} S\left(\delta^{R} S\right)$ the covectors identified by with coalgebra as follows

$$
\delta^{L} S(L)=\mathbb{L}(L) S, \quad \delta^{R} S(L)=\mathbb{R}(L) S .
$$

We will use $\delta$ for the left version. We can use the standard scalar product $(\cdot, \cdot)$ on bivectors to make the further identification of $\delta S$ with a bivector. ${ }^{6}$

For any function $S$ on the group, we can now define a Lagrangian submanifold

$$
\mathcal{L}_{S}=\{\theta=d S\}=\left\{p^{L}=\delta S\right\}=\left\{p^{R}=\delta^{R} S\right\}
$$

\footnotetext{
${ }^{6}$ This introduces additional factor of 2 in comparison with [8] (see section A).
} 


\subsection{Symplectic Theory of a Coadjoint Orbit}

Let us recall that we can identify the space of bivectors (Lie algebra $s o(1,3)=$ $\Lambda^{2} \mathbb{R}^{4}$ ) with the coalgebra using the natural scalar product $(\cdot, \cdot)$ on bivectors. Let us consider a coadjoint orbit

$$
X_{n, \rho}=\left\{B \in \Lambda^{2} \mathbb{R}^{4}:(B, B)=\frac{1}{4}\left(n^{2}-\rho^{2}\right),(B, * B)=-\frac{1}{2} \rho n\right\},
$$

where $C_{1}=(B, B)$ and $C_{2}=(B, * B)$ are two Casimirs (invariants). The Lorentz group acts transitively on $X_{n, \rho}$. We have a natural Poisson bracket given, for a linear function $H(L)(B)=(B, L)$, by

$$
\left\{H(L), H\left(L^{\prime}\right)\right\}=-H\left(\left[L, L^{\prime}\right]\right) .
$$

This turns the coadjoint orbits into symplectic manifolds. Let us introduce an isomorphism from $s o(1,3)$ to $\operatorname{sl}(2, \mathbb{C})$ (traceless matrices) by (see "Appendix A")

$$
B \rightarrow \mathbb{M}(B), \quad \mathbb{M}\left(v \wedge v^{\prime}\right)=\frac{1}{4}\left(\eta^{-}(v) \eta^{+}\left(v^{\prime}\right)-\eta^{-}\left(v^{\prime}\right) \eta^{+}(v)\right) .
$$

We have identity

$$
-2 \operatorname{tr} \mathbb{M}(B)^{2}=(B, B)-i(B, * B),
$$

thus for $B \in X_{n, \rho}$

$$
\frac{1}{2} \operatorname{tr} \mathbb{M}(B)^{2}=\left(\frac{1}{4}(\rho-i n)\right)^{2} .
$$

For the matrix $\mathbb{M}(B)$, there exist two spinors $\mathbb{Z}_{B}^{ \pm}$(unique up to a constant each) such that

$$
\mathbb{M}(B) \mathbb{Z}_{B}^{ \pm}= \pm \frac{1}{4}(\rho-i n) \mathbb{Z}_{B}^{ \pm}
$$

We can thus define a projection

$$
\pi: X_{n, \rho} \rightarrow \mathbb{C P}, \pi(B)=\left[\mathbb{Z}_{B}^{+}\right]
$$

Definition 3. A function

$$
S: U \subset \mathbb{C}^{2} \backslash\{0\} \rightarrow \mathbb{C} \text { modulo } 2 \pi
$$

is of type $(n, \rho)$ if

$$
S\left(r e^{i \phi} \mathbb{Z}\right)=S(\mathbb{Z})+\rho \ln r+n \phi .
$$

Usually we cannot define such actions globally. Let us introduce the no$\operatorname{tation}^{7}$ (where the action is on Weyl spinors $\mathcal{S}^{+}$, see "Appendix A")

$$
\delta_{\mathbb{Z}} f(\mathbb{Z})=\left.\delta_{g}^{L} f\left(g^{-1} \mathbb{Z}\right)\right|_{g=1} .
$$

Let us notice that $\delta_{\mathbb{Z}} S$ is a well-defined function on $\mathbb{C P}$ if $S$ satisfies (78).

For a given $\mathbb{Z} \in \mathbb{C}^{2} \backslash\{0\}$, we can consider a group $H_{[\mathbb{Z}]} \subset \mathrm{SL}(2, \mathbb{C})$ that preserves $[\mathbb{Z}] \in \mathbb{C P}$. The Lie algebra of this group is given by

$$
\text { Lie } H_{[\mathbb{Z}]}=\{B:[\mathbb{Z}, \mathbb{M}(B) \mathbb{Z}]=0\},
$$

\footnotetext{
${ }^{7}$ Our notation differs from [8].
} 
where $[\mathfrak{u}, \mathbb{v}]=\mathbb{u}^{T} \omega \mathbb{v}$ (see "Appendix A"). The subgroup that preserves $\mathbb{Z}$ is $H_{[\mathbb{Z}]}^{0}$

$$
\text { Lie } H_{[\mathbb{Z}]}^{0}=\left\{B: \mathbb{M}(B)_{\mathbb{Z}}=0\right\}=\left\{B: \exists \lambda \in \mathbb{C}, \mathbb{M}(B)=\lambda \mathbb{Z Z}^{T} \omega\right\}
$$

Let us notice that $B \in \operatorname{Lie} H_{[\mathbb{Z}]}^{0 \perp}$ is equivalent to

$$
\forall \lambda \in \mathbb{C}: 0=\Re \operatorname{tr} \mathbb{M}(B) \lambda \mathbb{Z Z}^{T} \omega=\Re \lambda[\mathbb{Z}, \mathbb{M}(B) \mathbb{Z}],
$$

thus to $B \in \operatorname{Lie} H_{[\mathbb{Z}]}$. Moreover, the scalar product is null on Lie $H_{[\mathbb{Z}]}^{0}$.

The functions of type $(0,0)$ are special as they can be pushed forward to $\mathbb{C P}$. For such $f$ we will denote $[f]_{\mathbb{C P}}$ such push forward, thus

$$
f=[f]_{\mathbb{C P}} \pi \text {. }
$$

We have the action of $\mathrm{SL}(2, \mathbb{C})$ on $\mathcal{S}^{+}$(and on $\mathbb{C P}$, respectively) generated by vector fields $\mathbb{L}_{\mathcal{S}^{+}}(L)\left(\mathbb{L}_{\mathbb{C P}}(L)\right.$, respectively) for $L \in s o(1,3)$. Vector fields $\mathbb{L}_{S^{+}}(L)$ (respectively, $\mathbb{L}_{\mathbb{C P}}(L)$ ) correspond to the jet of the curves

$$
t \rightarrow e^{-t L} \mathbb{Z}, \quad\left(t \rightarrow\left[e^{-t L} \mathbb{Z}\right], \text { respectively }\right) .
$$

Let us consider a map

$$
T_{[\mathbb{Z}]}^{*} \mathbb{C P} \ni p \rightarrow \phi_{[\mathbb{Z}]}(p) \in s o(1,3), \quad \forall_{L}\left(\phi_{[\mathbb{Z}]}(p), L\right)=p\left(\mathbb{L}_{\mathbb{C P}}(L)\right) \text { at point }[\mathbb{Z}] .
$$

Let us notice that for $f \in C^{\infty}(\mathbb{C P})$ we have

$$
\delta_{\mathbb{Z}}(f \pi)(\mathbb{Z})=\phi_{[\mathbb{Z}]}(d f) .
$$

Lemma 9. The map $\phi_{[\mathbb{Z}]}$ is a bijection from $T_{[\mathbb{Z}]}^{*} \mathbb{C P}$ to Lie $H_{[\mathbb{Z}]}^{0}$.

Proof. Let us notice that $\mathbb{L}_{\mathbb{C P}}(L)([\mathbb{Z}])=0$ if and only if $L \in$ Lie $H_{[\mathbb{Z}]}$ thus $\phi_{[\mathbb{Z}]} \in$ Lie $H_{[\mathbb{Z}]}^{\perp}=\operatorname{Lie} H_{[\mathbb{Z}]}^{0}$. As $s o(1,3)_{\mathbb{C P}}$ span the whole tangent space at $[\mathbb{Z}]$, we have also injectivity.

Lemma 10. For $S$ of type $(n, \rho)$, we have

$$
\delta_{\mathbb{Z}} S(\mathbb{Z}) \in \operatorname{Lie} H_{[\mathbb{Z}]} \cap X_{n, \rho}
$$

and $\pi\left(\delta_{\mathbb{Z}} S(\mathbb{Z})\right)=[\mathbb{Z}]$.

Proof. For any $L \in \operatorname{Lie} H_{[\mathbb{Z}]}^{0}$, we have $\delta_{\mathbb{Z}} S(L)=0$ thus $\delta_{\mathbb{Z}} S \in$ Lie $H_{[\mathbb{Z}]}^{0} \stackrel{\perp}{=}=$ Lie $H_{[\mathbb{Z}]}$. Let us consider a traceless matrix (for some spinor $u$ )

$$
\mathbb{N}=\frac{1}{2}\left(\mathfrak{u} \mathbb{Z}^{T}+\mathbb{Z u}^{T}\right) \omega,
$$

then $\mathbb{N}_{\mathbb{Z}}=\frac{1}{2}[u, \mathbb{Z}]_{\mathbb{Z}}$. Let furthermore $\mathbb{M}(B)=\mathbb{N}$, then we have

$$
\left(\delta_{\mathbb{Z}} S, B\right)=-\frac{1}{2}(\Re[u, \mathbb{Z}]) \rho-\frac{1}{2}(\Im[u, \mathbb{Z}]) n=-\Re \frac{1}{2}(\rho-i n)[\mathfrak{u}, \mathbb{Z}] .
$$

As $\mathbb{M}\left(\delta_{\mathbb{Z}} S\right) \in$ Lie $H_{[\mathbb{Z}]}$, we can write

$$
\mathbb{M}\left(\delta_{\mathbb{Z}} S\right)=\frac{1}{2}\left(\mathbb{v}^{T}+\mathbb{Z} \mathbb{V}^{T}\right) \omega
$$


and

$$
\left(\delta_{\mathbb{Z}} S, B\right)=-2 \operatorname{tr} \mathbb{M}\left(\delta_{\mathbb{Z}} S\right) \mathbb{N}=-[\mathbb{v}, \mathbb{Z}][\mathbb{u}, \mathbb{Z}],
$$

so $[\mathbb{v}, \mathbb{Z}]=\frac{1}{2}(\rho-i n)$ and as

$$
\mathbb{M}\left(\delta_{\mathbb{Z}} S\right)_{\mathbb{Z}}=\frac{1}{2}(\rho-i n)_{\mathbb{Z}}, \quad 2 \operatorname{tr} \mathbb{M}\left(\delta_{\mathbb{Z}} S\right)^{2}=[\mathbb{v}, \mathbb{Z}]^{2}=\frac{1}{4}(\rho-i n)^{2},
$$

thus $\delta_{\mathbb{Z}} S \in X_{n, \rho}$ and $\pi(\delta S)=[\mathbb{Z}]$.

Lemma 11. For any real function $S$ of type $(n, \rho)$, the map

$$
([\mathbb{Z}], p) \rightarrow \delta_{\mathbb{Z}} S+\phi_{[\mathbb{Z}]}(p)
$$

is a symplectic diffeomorphism from $T^{*} \mathbb{C P}$ to $X_{n, \rho}$. This map is compatible with the projection onto $\mathbb{C P}$.

Proof. Let us choose $f \in C^{\infty}(\mathbb{C P})$ such that $d f([\mathbb{Z}])=p$ then

$$
\delta_{\mathbb{Z}} S+\phi_{[\mathbb{Z}]}(p)=\delta_{\mathbb{Z}}(S+f \pi) \in X_{n, \rho},
$$

as $S+f \pi$ is of type $(n, \rho)$. Moreover,

$$
\mathbb{M}\left(\delta_{\mathbb{Z}}(S+f \pi)\right)(\mathbb{Z})=\frac{1}{4}(\rho-i n) \mathbb{Z}+0 \mathbb{Z},
$$

thus $\pi\left(\delta_{\mathbb{Z}}(S+f \pi)\right)=[\mathbb{Z}]$, so it is compatible with the projection on $\mathbb{C P}$.

If $B \in X_{n, \rho}$ and $\pi(B)=[\mathbb{Z}]$, then

$$
B-\delta_{\mathbb{Z}} S(\mathbb{Z}) \in \operatorname{Lie} H_{[\mathbb{Z}]}^{\perp}=\operatorname{Lie} H_{[\mathbb{Z}]}^{0} .
$$

However, $\phi_{[\mathbb{Z}]}$ is a bijection onto Lie $H_{[\mathbb{Z}]}^{0}$.

In order to check that it is a symplectomorphism, we will show that Poisson brackets between generators of $s o(1,3)$ are right. For $L \in s o(1,3)$ let us consider the pull back of the Hamiltonian $H(L)$ to $T^{*} \mathbb{C P}$. It is

$$
\delta_{\mathbb{Z}} S(\mathbb{Z})(L)+\theta\left(L_{\mathbb{C P}}\right)(\mathbb{Z})=\mathbb{L}_{\mathcal{S}^{+}}(S)(\mathbb{Z})+\theta\left(\mathbb{L}_{\mathbb{C P}^{P}}(L)\right)(\mathbb{Z}) .
$$

Let us notice that $\mathbb{L}_{\mathcal{S}^{+}}(S)$ descents to a function $\left[\mathbb{L}_{\mathcal{S}^{+}}(S)\right]_{\mathbb{C P}}$ on $\mathbb{C P}$. We have thus for a given bivector a function on $T^{*} \mathbb{C P}$

$$
H_{\mathbb{C P}}(L)=\left[\mathbb{L}_{\mathcal{S}^{+}}(L)(S)\right]_{\mathbb{C P}}+\theta\left(\mathbb{L}_{\mathbb{C P}}(L)\right) .
$$

Let us notice that

$$
\begin{gathered}
\left\{\left[\mathbb{L}_{\mathcal{S}}(L)(S)\right]_{\mathbb{C P}},\left[\mathbb{L}_{\mathcal{S}^{+}}\left(L^{\prime}\right)(S)\right]_{\mathbb{C P}}\right\}=0, \\
\left\{\theta\left(\mathbb{L}_{\mathbb{C P}}(L)\right), \theta\left(\mathbb{L}_{\mathbb{C P}}\left(L^{\prime}\right)\right)\right\}=-\theta\left(\mathbb{L}_{\mathbb{C P}}\left(\left[L, L^{\prime}\right]\right)\right) .
\end{gathered}
$$

Moreover,

$$
\left\{\left[\mathbb{L}_{\mathcal{S}^{+}}(L)(S)\right]_{\mathbb{C P}}, \theta\left(\mathbb{L}_{\mathbb{C P}^{P}}\left(L^{\prime}\right)\right)\right\}=\left[\mathbb{L}_{\mathcal{S}^{+}}\left(L^{\prime}\right) \mathbb{L}_{\mathcal{S}^{+}}(L)(S)\right]_{\mathbb{C P}},
$$

thus

$$
\begin{aligned}
& \left\{\left[\mathbb{L}_{\mathcal{S}^{+}}(L)(S)\right]_{\mathbb{C P}}, \theta\left(\mathbb{L}_{\mathbb{C P}}\left(L^{\prime}\right)\right)\right\}-\left\{\left[\mathbb{L}_{\mathcal{S}^{+}}\left(L^{\prime}\right)(S)\right]_{\mathbb{C P}}, \theta\left(\mathbb{L}_{\mathbb{C P}^{P}}(L)\right)\right\} \\
& \quad=\left[\mathbb{L}_{\mathcal{S}^{+}}\left(\left[L^{\prime}, L\right]\right)(S)\right]_{\mathbb{C P}} .
\end{aligned}
$$

Therefore, finally

$$
\left\{H(L), H\left(L^{\prime}\right)\right\}=-H\left(\left[L, L^{\prime}\right]\right) .
$$


Because the Hamiltonian vector fields of functions span in every point the whole tangent space, $\Omega$ is the same as the canonical symplectic form on the cotangent bundle.

Let us now consider a complex action (locally defined) $S$ of type $(n, \rho)$. Let us notice that $\Im S$ is a function on $\mathbb{C P}$. In particular, $\partial^{2}[\Im S]_{\mathbb{C P}}$ is a tensor on $\mathbb{C P}$.

Lemma 12. The space

$$
\mathcal{L}_{S}^{\prime}=\left\{\delta_{\mathbb{Z}} S:[\mathbb{Z}] \in U\right\}
$$

is a complex Lagrangian manifold in $X_{n, \rho}$ and on the real point $B \in X_{n, \rho}$

$$
I_{B}\left(v, v^{\prime}\right)=\partial^{2}[\Im S([\mathbb{Z}])]_{\mathbb{C P}}\left(\overline{\pi(v)}, \pi\left(v^{\prime}\right)\right),
$$

where $v \in T \mathcal{L}_{S}^{\prime}$ and $[\mathbb{Z}]=\pi(B)$.

Remark. We regard $\mathbb{C P}$ as a real manifold, thus $\pi(v) \in T^{\mathbb{C}} \mathbb{C P}$ and the conjugation is with respect to this additional complex structure. It can be translated into inner complex conjugation.

Proof. Let $S_{a u x}$ be an auxiliary real action of type $(n, \rho)$. The difference $f=S-S_{a u x}$ is a well-defined function on $\mathbb{C P}$. Moreover, using the local identification of $X$ with $\mathbb{C P}$, we have

$$
\mathcal{L}_{S}^{\prime}=\left\{\theta=d[f]_{\mathbb{C P}}\right\}
$$

Indeed this is equivalent to

$$
B=\delta S_{a u x}+\phi_{[\mathbb{Z}]}(p)=\delta S_{a u x}+\phi_{[\mathbb{Z}]}\left(d[f]_{\mathbb{C P}}\right)=\delta S_{a u x}+\delta f=\delta S .
$$

We know that

$$
\partial^{2}[\Im S]_{\mathbb{C P}}\left(\overline{\pi(v)}, \pi\left(v^{\prime}\right)\right)=\partial^{2}\left[\Im\left(S-S_{a u x}\right)\right]_{\mathbb{C P}}\left(\overline{\pi(v)}, \pi\left(v^{\prime}\right)=I\left(v, v^{\prime}\right),\right.
$$

thus the result.

\subsection{Casimir Reduction}

Let us consider a symplectic reduction of $T^{*} \mathrm{SL}(2, \mathbb{C})$ with respect to Casimirs. For $\mathrm{SL}(2, \mathbb{C})$ the moment map is nondegenerate except for bivectors equal to zero.

Lemma 13. Two points $(g, p)$ and $\left(g^{\prime}, p^{\prime}\right)$ are connected by a flow of Casimirs in $\mathrm{SL}(2, \mathbb{C})$ if and only if there exists $\lambda, \lambda^{\prime} \in \mathbb{R}$ such that

$$
g=g^{\prime} e^{\lambda p+\lambda^{\prime} * p}
$$

and $p^{L}=p^{\prime L} \quad\left(\right.$ or equivalently $\left.p^{R}=p^{\prime R}\right)$.

Proof. Left covectors are preserved by Casimirs; thus, we only need to find the vector field on the group. Let us denote the projection on the group manifolds of the Poisson vector fields of the Casimirs by $V_{1}$ and $V_{2}$.

We identify bivectors with the left covectors on $\mathrm{SL}(2, \mathbb{C})$ by the scalar product and then

$$
V_{1}=2 \mathbb{L}\left(p^{L}\right),
$$


and thus $g$ is changed from the right (because left invariant vector field) by $p^{L}$.

The second Casimir is related to the first by Hodge star, thus

$$
V_{2}=2 \mathbb{L}\left(* p^{L}\right) .
$$

Together (they commute) we have the flow

$$
g^{\prime}=g e^{\lambda p^{L}+\lambda^{\prime} * p^{L}} .
$$

From preservation of left covectors, we have $p^{L}=p^{\prime L}$.

The symplectic reduction with respect to the Casimirs is given by

$$
([g], B): B \in X_{n, \rho}, \quad[g]=\left[g^{\prime}\right] \text { if } \exists_{\lambda, \lambda^{\prime} \in \mathbb{R}} g=g^{\prime} e^{\lambda B+\lambda^{\prime} * B} .
$$

Let us denote

$$
C_{n, \rho}=\left\{(g, B): B \in X_{n, \rho}\right\} \subset T^{*} \mathrm{SL}(2, \mathbb{C}) .
$$

We have a map $\pi_{C_{n, \rho}}: C_{n, \rho} \rightarrow S$ to the symplectic reduction.

If $\mathcal{L}^{\prime} \subset S$ is a real Lagrangian, then

$$
\pi_{C_{n, \rho}}^{-1}\left(\mathcal{L}^{\prime}\right)
$$

is also a Lagrangian and it is a subset of $C_{n, \rho}$. The other way around, if a real Lagrangian $\mathcal{L} \subset T^{*} \mathrm{SL}(2, \mathbb{C})$ is such that $\mathcal{L} \subset C_{n, \rho}$, then as Casimir generated directions belong to $\mathcal{L}$ we have

$$
\mathcal{L}=\pi_{C_{n, \rho}}^{-1}\left(\mathcal{L}^{\prime}\right)
$$

where $\mathcal{L}^{\prime}$ is a Lagrangian in $S$.

The same holds for complex Lagrangians (in locally holomorphic extensions).

4.3.1. Explicit Description. There is a direct description of this symplectic reduction that is an analog of Peter-Weyl theorem in group representation theory. Let us notice that the left and right invariant covectors Poisson commute with the Casimirs. Moreover, the equation

$$
p^{R}=-g^{-1} \cdot p^{L}
$$

has a solution for $g$ if $p^{R}$ and $p^{L}$ are of the same type (nonzero) and $g$ is unique up to [.] equivalence. Thus the map

$$
([g], p) \rightarrow\left(p^{L}, p^{R}\right) \in X_{n, \rho} \times X_{n, \rho}
$$

is an isomorphism of symplectic spaces. ${ }^{8}$

\footnotetext{
${ }^{8}$ We used the fact that if $B \in X_{n, \rho}$, then also $-B \in X_{n, \rho}$.
} 


\subsection{Symplectic Theory of $S_{i j}^{\prime}$}

Let us consider an action

$$
\tilde{S}_{i j}\left(g_{i}, g_{j}, \mathbb{Z}_{i j}, \mathbb{Z}_{j i}\right)=S_{i j}^{n_{i j}}\left(g_{i}^{-1} \mathbb{Z}_{i j}\right)+S_{i j}^{\beta}\left(\mathbb{Z}_{i j}, \mathbb{Z}_{j i}\right)+S_{j i}^{n_{j i}}\left(g_{j}^{-1} \mathbb{Z}_{j i}\right) .
$$

Let us now assume that for every $i j$ the Lagrangian

$$
\mathcal{L}_{i j}^{\prime}=\mathcal{L}_{S_{i j}^{n_{i j}}}^{\prime} \subset X_{2 j_{i j}, \rho_{i j}}
$$

is strictly positive at the point corresponding to the fundamental stationary point (that is $\left.\left[\left(g_{i}^{0}\right)^{-1} \mathbb{Z}_{i j}^{0}\right]\right)$. We will prove this fact in Sect. 5.3.

Because the action $S^{\beta}$ is real, the imaginary part of the Hessian with respect to $\mathbb{Z}_{i j}$ and $\mathbb{Z}_{i j}$ is block diagonal with respect to every $\mathbb{Z}$ variable. From strict positivity of the Lagrangian, every block is strictly positive; thus, by Lemma 1 , the form $H_{\mathbb{Z Z}}$ is nondegenerate.

We can now consider

$$
S_{i j}^{\mathrm{red}}\left(g_{i}, g_{j}\right)=S_{i j}^{\prime}\left(g_{i j}\right) .
$$

It is well defined for $g_{i j}$ in the neighbourhood of $g_{i j}^{0}$.

Lemma 14. The Lagrangian manifold of the action $S_{i j}^{\prime}$ is given by

$$
\mathcal{L}_{S_{i j}^{\prime}}=\pi_{C_{2 j_{i j}, \rho_{i j}}}^{-1}\left(\mathcal{L}_{i j}^{\prime} \times \mathcal{L}_{j i}^{\prime}\right) .
$$

Proof. Left and right invariant derivatives of $S_{i j}^{\prime}$ are equal to derivatives of $S_{i j}^{n_{i j}}$ and, respectively, $S_{j i}^{n_{j i}}$ with spinors equal to the stationary point solutions $\mathbb{Z}^{\mathbb{C}}$

$$
\delta^{L} S_{i j}^{\prime}=\delta_{g_{i}}^{L} S_{i j}^{\mathrm{red}}=\delta_{\mathbb{Z}} S_{i j}^{n_{i j}}\left(g_{i}^{-1} \mathbb{Z}_{i j}^{\mathbb{C}}\right), \quad \delta^{R} S_{i j}^{\prime}=\delta_{g_{j}}^{L} S_{i j}^{\mathrm{red}}=\delta_{\mathbb{Z}} S_{j i}^{n_{i j}}\left(g_{j}^{-1} \mathbb{Z}_{j i}^{\mathbb{C}}\right),
$$

because derivatives with respect to $\mathbb{Z}$ vanish in the point $\left[\mathbb{Z}^{\mathbb{C}}\right]\left(g_{i}, g_{j}\right)$. We see from the type of the actions that $\mathcal{L}_{S_{i j}^{\prime}} \subset C_{2 \jmath_{i j} \rho_{i j}}^{\mathbb{C}}$; thus, it is an inverse image of a complex Lagrangian in $X_{2 \jmath_{i j}, \rho_{i j}} \times X_{2 \jmath_{i j}, \rho_{i j}}$. We see also that

$$
\pi_{C_{2 j_{i j} \rho_{i j}}}\left(\mathcal{L}_{S_{i j}^{\prime}}\right) \subset \mathcal{L}_{i j}^{\prime} \times \mathcal{L}_{j i}^{\prime}
$$

and by comparing dimension it needs to be equal.

Let us denote

$$
B_{i j}^{0^{\prime}}=\delta_{\mathbb{Z}} S_{i j}^{n_{i j}}\left(\left(g_{i}^{0}\right)^{-1} \mathbb{Z}_{i j}^{0}\right) .
$$

Let us notice $B_{i j}^{0^{\prime}}=\left(g_{i}^{0}\right)^{-1} B_{i j}^{0}$.

Lemma 15. If every Lagrangian $\mathcal{L}_{i j}^{\prime}$ is strictly positive, then if for $v \in \operatorname{so}(1,3)$

$$
\left(\partial^{2} \Im S_{i j}^{\prime}\right) v=0
$$

then $v \in\left\{B_{i j}^{0^{\prime}}, * B_{i j}^{0^{\prime}}\right\}$.

Proof. From the previous lemma

$$
\mathcal{L}_{S_{i j}^{\prime}}=\pi_{C_{2 \jmath_{i j}, \rho_{i j}}}^{-1}\left(\mathcal{L}_{i j}^{\prime} \times \mathcal{L}_{j i}^{\prime}\right) .
$$

Let $V=\Pi_{S_{i j}^{\prime}}^{-1}(v)$ be the lift of $v$ to $T \mathcal{L}_{S_{i j}^{\prime}}$, its image

$$
\pi_{C_{2 j_{i j}, \rho_{i j}}}(V) \in T\left(\mathcal{L}_{i j}^{\prime} \times \mathcal{L}_{j i}^{\prime}\right) \cap \overline{T\left(\mathcal{L}_{i j}^{\prime} \times \mathcal{L}_{j i}^{\prime}\right)}=\{0\} .
$$


Thus $V$ is in the space of the Casimirs' Poisson vector fields. Thus its projection onto the tangent space of the group

$$
v \in\left\{B_{i j}^{0^{\prime}}, * B_{i j}^{0^{\prime}}\right\}
$$

as stated.

\section{Simplicity Constraints}

Our goal in this section is to show that $\mathcal{L}_{i j}^{\prime}$ is strictly positive at the extremal point coming from the fundamental stationary point. In fact it is a simple computation of a two-dimensional matrix. However, it is useful to describe this Lagrangian (in the neighbourhood of this point). Let us notice that from the reality condition of the action, we know that the Lagrangian is positive.

\subsection{Conditions on the Action}

Let us suppose that we have a function of the form

$$
G^{N}(\mathbb{Z})=f(\mathbb{Z}) e^{i N S(\mathbb{Z})},
$$

defined and analytic for $\mathbb{Z} \in U$.

We have an action of the group on spinors $\mathbb{Z}$; thus, we can also consider an operator

$$
\hat{D}=\sum_{|I| \leq m}(-i)^{|I|} d_{|I|}^{I_{1} \ldots I_{|I|}} \mathbb{L}_{\mathcal{S}^{+}}\left(L_{I_{1}}\right) \ldots \mathbb{L}_{\mathcal{S}^{+}}\left(L_{I_{|I|}}\right),
$$

where $L_{I}$ are Lie algebra basis.

We associate with this operator a symbol (a homogenous polynomial on the Lie coalgebra)

$$
P_{D}(p)=\sum_{|I|=m} d_{m}^{I_{1} \ldots I_{m}} L_{I_{1}}(p) \ldots L_{I_{m}}(p),
$$

where $p$ are Lie coalgebra elements. Let us remind that we identify both Lie algebra and coalgebra with bivectors (thanks to the scalar product).

Let $p(\lambda)$ be a polynomial of order $m$ with $m$-homogeneous coefficient $a_{m}$ such that for every $N$

$$
(\hat{D}-p(N)) G^{N}(\mathbb{Z})=0 .
$$

Then taking the leading term in the $N$ expansion, we get for any $\mathbb{Z}$

$$
P_{D}\left(\delta_{\mathbb{Z}} S\right)=a_{m}
$$

\subsection{Bivector Decomposition}

For the given normal $N_{i}^{0}$ (see [8]) with the norm $c_{i}=\left|N_{i}^{0}\right|^{2} \in\{-1,1\}$, we can decompose the bivector $B$ as follows

$$
B=*\left(v \wedge N_{i}^{0}\right)+w \wedge N_{i}^{0},
$$

where $v, w \in{N_{i}^{0}}^{\perp}$ and the two terms belong to

$$
s o\left(N_{i}^{0 \perp}\right) \oplus * s o\left(N_{i}^{0 \perp}\right) .
$$


We can now introduce maps

$$
\begin{array}{ll}
\overrightarrow{\mathbb{L}}_{i}: s o(1,3) \rightarrow N_{i}^{0 \perp}, & \overrightarrow{\mathbb{L}}_{i}(B)=v, \\
\overrightarrow{\mathbb{K}}_{i}: s o(1,3) \rightarrow N_{i}^{0 \perp}, & \overrightarrow{\mathbb{K}}_{i}(B)=w .
\end{array}
$$

They are explicitly given by

$$
\overrightarrow{\mathbb{L}}_{i}(B)=c_{i} N_{i}^{0}\left\llcorner * B, \quad \overrightarrow{\mathbb{K}}_{i}(B)=-c_{i} N_{i}^{0}\llcorner B .\right.
$$

We can identify $s o\left(N_{i}^{0 \perp}\right)$ with the vector space $N_{i}^{0^{\perp}}$ by the map $\overrightarrow{\mathbb{L}}_{i}$

$$
\left[*\left(v \wedge N_{i}^{0}\right), *\left(v^{\prime} \wedge N_{i}^{0}\right)\right]=*\left(\left(v \times v^{\prime}\right) \wedge N_{i}^{0}\right),
$$

where $x$ is defined by

$$
v \times v^{\prime}=*\left(v \wedge N_{i}^{0}\right)\left(v^{\prime}\right)=-*\left(v \wedge v^{\prime} \wedge N_{i}^{0}\right) .
$$

The Casimirs can be written in terms of these vectors as follows

$$
C_{1}=(B, B)=-c_{i}\left(\overrightarrow{\mathbb{L}}_{i}^{2}-\overrightarrow{\mathbb{K}}_{i}^{2}\right), \quad C_{2}=(B, * B)=-2 c_{i} \overrightarrow{\mathbb{L}}_{i} \cdot \overrightarrow{\mathbb{K}}_{i}
$$

With the vector $v \in N_{i}^{0^{\perp}}$ we can associate two complex vectors $k_{s}^{i}(v)(s= \pm 1)$ given by the conditions:

1. $k_{s}^{i}(v) \cdot N_{0}^{i}=k_{s}^{i}(v) \cdot v=k_{s}^{i}(v) \cdot k_{s}^{i}(v)=0$.

2. The action of the vector on $k_{s}$

$$
\begin{gathered}
v \times k_{s}^{i}(v)=i s C k_{s}^{i}(v), \\
\text { where } C=\sqrt{\left(*\left(v \wedge N_{i}^{0}\right), *\left(v \wedge N_{i}^{0}\right)\right)}=\sqrt{-c_{i} v \cdot v} .
\end{gathered}
$$

In the case of spacelike faces, we choose $C>0$. In this situation vectors $k_{ \pm 1}^{i}(v)$ are complex and we assume

$$
k_{-1}^{i}(v)=\overline{k_{1}^{i}(v)}
$$

With the choice of signature $(+---)$ the Hermitian form $\bar{w} \cdot w$ on $\left\{N_{i}^{0}, v\right\}^{\perp}$ is negatively definite, thus $k_{1}^{i}(v) \cdot k_{-1}^{i}(v)<0$. We assume that $k_{1}^{i}(v) \cdot k_{-1}^{i}(v)=-1$, and this fixes vectors up to a phase.

Lemma 16. We have

$$
k_{1}^{i}(v) \times k_{-1}^{i}(v)=i \frac{c_{i}}{C} v .
$$

Proof. Let us notice that $k_{1}^{i}(v) \times k_{-1}^{i}(v)=\alpha v$ and

$$
\begin{aligned}
& i C k_{-1}^{i}(v) \cdot k_{1}^{i}(v)=k_{-1}^{i}(v) \cdot\left(v \times k_{1}^{i}(v)\right) \\
& =-k_{-1}^{i}(v) \cdot\left(k_{1}^{i}(v) \times v\right)=\left(k_{1}^{i}(v) \times k_{-1}^{i}(v)\right) \cdot v=\alpha(v \cdot v) .
\end{aligned}
$$

Thus

$$
k_{1}^{i}(v) \times k_{-1}^{i}(v)=i \frac{C\left(k_{-1}^{i}(v) \cdot k_{1}^{i}(v)\right)}{v \cdot v} v,
$$

and substituting $v \cdot v=-c_{i} C^{2}$ we get the result. 

then

Let us notice that if a complex vector $w \in N_{i}^{0^{\perp}}$ satisfies $w \cdot w=w \cdot v=0$,

$$
w \in \operatorname{span}\left\{k_{1}^{i}(v)\right\} \cup \operatorname{span}\left\{k_{-1}^{i}(v)\right\} .
$$

We can regard $v \cdot \overrightarrow{\mathbb{L}}_{i}$ and $v \cdot \overrightarrow{\mathbb{K}}_{i}$ as linear maps on bivectors; thus, we can compute Poisson brackets. In order to do it, we need to find the associated by (the scalar product) bivectors

$$
v \cdot \overrightarrow{\mathbb{L}}_{i}(B)=\left(-c_{i} *\left(v \wedge N_{i}^{0}\right), B\right), \quad v \cdot \overrightarrow{\mathbb{K}}_{i}(B)=\left(c_{i} v \wedge N_{i}^{0}, B\right),
$$

thus we get

$$
\begin{aligned}
& \left\{v \cdot \overrightarrow{\mathbb{L}}_{i}, v^{\prime} \cdot \overrightarrow{\mathbb{L}}_{i}\right\}=c_{i}\left(v \times v^{\prime}\right) \cdot \overrightarrow{\mathbb{L}}_{i}, \\
& \left\{v \cdot \overrightarrow{\mathbb{L}}_{i}, v^{\prime} \cdot \overrightarrow{\mathbb{K}}_{i}\right\}=c_{i}\left(v \times v^{\prime}\right) \cdot \overrightarrow{\mathbb{K}}_{i}, \\
& \left\{v \cdot \overrightarrow{\mathbb{K}}_{i}, v^{\prime} \cdot \overrightarrow{\mathbb{K}}_{i}\right\}=-c_{i}\left(v \times v^{\prime}\right) \cdot \overrightarrow{\mathbb{L}}_{i} .
\end{aligned}
$$

\subsection{Simplicity Constraints}

The coherent states $\Phi^{n_{i j}}\left(\mathbb{Z}_{i j}\right)$ satisfy the following equations

1. Diagonal simplicity constraints, that for fixed spins means that the values of the Casimir operators are related to twisted simplicity constraints ${ }^{9}$

$$
\hat{C}_{1}=\frac{1}{4}\left(n^{2}-\rho^{2}-4\right), \hat{C}_{2}=-\frac{1}{2} n \rho,
$$

where $\rho=\gamma n$ and $n=2 \jmath_{i j} \cdot{ }^{10}$

2. Cross simplicity constraints, that are implemented in the EPRL model by

$$
\begin{aligned}
& \left(\gamma \hat{\vec{L}}_{i}+\hat{\vec{K}}_{i}\right)^{2}=0, \\
& \left(\hat{\vec{L}}_{i}-\gamma \hat{\vec{K}}_{i}\right) \cdot\left(\gamma \hat{\vec{L}}_{i}+\hat{\vec{K}}_{i}\right)=0 .
\end{aligned}
$$

3. The coherent state condition $k_{s_{i j}}\left(v_{i j}\right) \cdot \hat{\vec{L}}_{i}=0$, where $s_{i j}$ is fixed and $v_{i j}$ is constructed from $n_{i j}$.

These conditions impose several conditions on $S_{i j}^{n_{i j}}$. We can describe them in terms of $\mathcal{L}_{i j}^{\prime}$. Namely $B \in \mathcal{L}_{i j}^{\prime}$ needs to satisfy

1. Diagonal simplicity constraints $(B, B)=\frac{1}{4}\left(4 J_{i j}^{2}-\rho_{i j}^{2}\right)$ and $(B, * B)=$ $-\frac{1}{2} 2 \jmath_{i j} \rho_{i j}$ that are satisfied because $B \in X_{2 \jmath_{i j}, \rho_{i j}}$.

2. Cross simplicity constraints

$$
\begin{aligned}
& \left(\gamma \overrightarrow{\mathbb{L}}_{i}+\overrightarrow{\mathbb{K}}_{i}\right)^{2}=0, \\
& \left(\overrightarrow{\mathbb{L}}_{i}-\gamma \overrightarrow{\mathbb{K}}_{i}\right) \cdot\left(\gamma \overrightarrow{\mathbb{L}}_{i}+\overrightarrow{\mathbb{K}}_{i}\right)=0 .
\end{aligned}
$$

3. Coherent state condition $k_{s_{i j}}\left(v_{i j}\right) \cdot \overrightarrow{\mathbb{L}}_{i}=0$, where $s_{i j}$ is fixed and $v_{i j}$ is constructed from $n_{i j}$.

\footnotetext{
${ }^{9}$ Quantisation of the action of the Lie algebra element $L$ is $\hat{L}=\frac{1}{i} \mathbb{L}_{\mathcal{S}^{+}}(L)$.

${ }^{10}$ Our convention differs from [3] by a sign in $C_{2}$ that can be seen from (142).
} 
In order to analyse the constraints let us introduce a twisting map

$$
\tau: s o(1,3) \rightarrow s o(1,3), \quad \tau(B)=B+\gamma * B .
$$

We can compute

$$
\begin{gathered}
(\tau(B), \tau(B))=\left(1-\gamma^{2}\right)(B, B)+2 \gamma(B, * B), \\
(\tau(B), * \tau(B))=\left(1-\gamma^{2}\right)(B, * B)-2 \gamma(B, B) .
\end{gathered}
$$

Similarly

$$
\overrightarrow{\mathbb{L}}_{i}(\tau(B))=\overrightarrow{\mathbb{L}}_{i}(B)+\gamma \overrightarrow{\mathbb{K}}_{i}(B), \quad \overrightarrow{\mathbb{K}}_{i}(\tau(B))=\overrightarrow{\mathbb{K}}_{i}(B)-\gamma \overrightarrow{\mathbb{L}}_{i}(B) .
$$

Let us denote $B^{\tau}=\tau^{-1}(B)$ and $\overrightarrow{\mathbb{L}}_{i}^{\tau}(B)=\overrightarrow{\mathbb{L}}_{i}\left(B^{\tau}\right), \overrightarrow{\mathbb{K}}_{i}^{\tau}(B)=\overrightarrow{\mathbb{K}}_{i}\left(B^{\tau}\right)$, then

$$
\overrightarrow{\mathbb{L}}_{i}(B)=\overrightarrow{\mathbb{L}}_{i}^{\tau}(B)+\gamma \overrightarrow{\mathbb{K}}_{i}^{\tau}(B), \quad \overrightarrow{\mathbb{K}}_{i}(B)=\overrightarrow{\mathbb{K}}_{i}^{\tau}(B)-\gamma \overrightarrow{\mathbb{L}}_{i}^{\tau}(B) .
$$

The first two conditions mean

1. Diagonal simplicity conditions:

$$
\left(B^{\tau}, B^{\tau}\right)=j_{i j}^{2}, \quad\left(B^{\tau}, * B^{\tau}\right)=0 .
$$

2. Cross simplicity: $\overrightarrow{\mathbb{K}}_{i}^{\tau} \in \operatorname{span}\left\{k_{1}^{i}\left(\overrightarrow{\mathbb{L}}_{i}^{\tau}\right)\right\} \cup \operatorname{span}\left\{k_{-1}^{i}\left(\overrightarrow{\mathbb{L}}_{i}^{\tau}\right)\right\}$.

Thus we can write

$$
B^{\tau}=*\left(v \wedge N_{i}^{0}\right)+\lambda^{\prime} k_{t}^{i}(v) \wedge N_{i}^{0}
$$

and the Casimir conditions means that

$$
-c_{i}|v|^{2}=j_{i j}^{2}
$$

We are interested in the fundamental stationary point, and then $\left(B_{i j}^{0^{\prime}}\right)^{\tau}=$ $* v_{i j} \wedge N_{i}^{0}$. The space $\mathcal{L}_{i j}^{\prime}$ around this point is a manifold; thus, there is a choice $t_{i j}$ such that

$$
\overrightarrow{\mathbb{K}}_{i}^{\tau} \in \operatorname{span}\left\{k_{t_{i j}}^{i}\left(\overrightarrow{\mathbb{L}}_{i}^{\tau}\right)\right\}
$$

We also have

$$
-i\left\{\overline{k_{t_{i j}}^{i}(v)} \cdot\left(\overrightarrow{\mathbb{K}}_{i}+\gamma \overrightarrow{\mathbb{L}}_{i}\right), k_{t_{i j}}^{i}(v) \cdot\left(\overrightarrow{\mathbb{K}}_{i}+\gamma \overrightarrow{\mathbb{L}}_{i}\right)\right\}=-t_{i j} \frac{v}{C} \cdot\left(\left(\gamma^{2}-1\right) \overrightarrow{\mathbb{L}}_{i}+2 \gamma \overrightarrow{\mathbb{K}}_{i}\right),
$$

and, from positivity of the Lagrangian, the right-hand side needs to be positive. Let us notice that

$$
\frac{v}{C} \cdot\left(\left(\gamma^{2}-1\right) \overrightarrow{\mathbb{L}}_{i}+2 \gamma \overrightarrow{\mathbb{K}}_{i}\right)=\left(1+\gamma^{2}\right) \frac{v}{C} \cdot\left(\gamma \overrightarrow{\mathbb{K}}_{i}^{\tau}-\overrightarrow{\mathbb{L}}_{i}^{\tau}\right) .
$$

As at $B=B_{i j}^{0^{\prime}}$ we have $\left(C=\jmath_{i j}\right)$

$$
v_{i j} \cdot \overrightarrow{\mathbb{L}}_{i}^{\tau}=\left|v_{i j}\right|^{2}=-c_{i} C^{2}, \quad v_{i j} \cdot \overrightarrow{\mathbb{K}}_{i}^{\tau}=0,
$$

we see that $t_{i j}=-c_{i}$.

Let us consider now coherent state condition $k_{s_{i j}}\left(v_{i j}\right) \cdot \overrightarrow{\mathbb{L}}_{i}=0$. It means that

$$
\overrightarrow{\mathbb{L}}_{i}=\lambda_{1} v_{i j}+\lambda_{2} k_{s_{i j}}\left(v_{i j}\right)
$$

However,

$$
\overrightarrow{\mathbb{L}}_{i}^{2}=\left(\lambda_{1} v_{i j}+\lambda_{2} k_{s_{i j}}\left(v_{i j}\right)\right)^{2}=\lambda_{1}^{2}\left|v_{i j}\right|^{2} .
$$


but $\overrightarrow{\mathbb{L}}_{i}^{\tau} \cdot \overrightarrow{\mathbb{K}}_{i}^{\tau}=\overrightarrow{\mathbb{K}}_{i}^{\tau} \cdot \overrightarrow{\mathbb{K}}_{i}^{\tau}=0$, thus

$$
\overrightarrow{\mathbb{L}}_{i}^{2}=\left(\overrightarrow{\mathbb{L}}_{i}^{\tau}+\gamma \overrightarrow{\mathbb{K}}_{i}^{\tau}\right)^{2}=\left(\overrightarrow{\mathbb{L}}_{i}^{\tau}\right)^{2}=-c_{i}\left[-c_{i}\left(\left(\overrightarrow{\mathbb{L}}_{i}^{\tau}\right)^{2}-\left(\overrightarrow{\mathbb{K}}_{i}^{\tau}\right)^{2}\right)\right]=-c_{i} j_{i j}^{2},
$$

and $\lambda_{1}= \pm 1$. As the phase space point corresponding to the fundamental stationary point is in the Lagrangian we have in the neighbourhood of this stationary point $\lambda_{1}=1$. We can now compute

$$
-i\left\{k_{-s_{i j}}\left(v_{i j}\right) \cdot \overrightarrow{\mathbb{L}}_{i}, k_{s_{i j}}\left(v_{i j}\right) \cdot \overrightarrow{\mathbb{L}}_{i}\right\}=-s_{i j} \frac{v_{i j}}{C} \cdot \overrightarrow{\mathbb{L}}_{i}=-s_{i j} \frac{v_{i j}}{C} \cdot\left(\overrightarrow{\mathbb{L}}_{i}^{\tau}+\gamma \overrightarrow{\mathbb{K}}_{i}^{\tau}\right) \text {, }
$$

at the fundamental stationary point it is equal to $s_{i j} c_{i} \jmath_{i j}$, thus $s_{i j}=c_{i}$.

We can now describe tangent space to the Lagrangian at $B_{i j}^{0^{\prime}}$. The conditions for bivectors to be tangent directions to $\mathcal{L}_{i j}^{\prime}$ are that

1. Tangency condition $\left(B, B_{i j}^{0^{\prime}}\right)=0,\left(B, * B_{i j}^{0^{\prime}}\right)=0$ (this is equivalent to $v_{i j} \cdot \overrightarrow{\mathbb{L}}_{i}(B)=v_{i j} \cdot \overrightarrow{\mathbb{K}}_{i}(B)=0$ and also $\left.v_{i j} \cdot \overrightarrow{\mathbb{L}}_{i}^{\tau}(B)=v_{i j} \cdot \overrightarrow{\mathbb{K}}_{i}^{\tau}(B)=0\right)$,

2. $k_{t_{i j}}\left(v_{i j}\right) \cdot\left(\overrightarrow{\mathbb{K}}_{i}(B)+\gamma \overrightarrow{\mathbb{L}}_{i}(B)\right)=0$ (that is $\left.k_{t_{i j}}\left(v_{i j}\right) \cdot \overrightarrow{\mathbb{K}}_{i}^{\tau}(B)=0\right)$,

3. $k_{s_{i j}}\left(v_{i j}\right) \cdot \overrightarrow{\mathbb{L}}_{i}(B)=0$.

It is not hard to find all vectors satisfying these conditions. Every tangent bivector can be uniquely described by a pair

$$
B \rightarrow\left(\overrightarrow{\mathbb{L}}_{i}(B), \overrightarrow{\mathbb{K}}_{i}^{\tau}(B)\right) .
$$

The conditions on $B$ are

$$
\left(\overrightarrow{\mathbb{L}}_{i}(B), \overrightarrow{\mathbb{K}}_{i}^{\tau}(B)\right)=\left(\lambda_{s} k_{s_{i j}}\left(v_{i j}\right), \lambda_{t} k_{t_{i j}}\left(v_{i j}\right)\right), \quad \lambda_{s}, \lambda_{t} \in \mathbb{C} .
$$

We can now summarize

Lemma 17. At the point $B_{i j}^{0^{\prime}}$ the Lagrangian $\mathcal{L}_{i j}^{\prime}$ is strictly positive.

Proof. We need to prove that the real tangent vector (bivector) is zero. Tangent vectors satisfy

$$
\left(\overrightarrow{\mathbb{L}}_{i}(B), \overrightarrow{\mathbb{K}}_{i}^{\tau}(B)\right)=\left(\lambda_{s} k_{s_{i j}}\left(v_{i j}\right), \lambda_{t} k_{t_{i j}}\left(v_{i j}\right)\right), \quad \lambda_{s}, \lambda_{t} \in \mathbb{C},
$$

and from reality

$$
\begin{aligned}
& \left(\overrightarrow{\mathbb{L}}_{i}(B), \overrightarrow{\mathbb{K}}_{i}^{\tau}(B)\right)=\left(\overrightarrow{\mathbb{L}}_{i}(\bar{B}), \overrightarrow{\mathbb{K}}_{i}^{\tau}(\bar{B})\right) \\
& \quad=\left(\overline{\lambda_{s} k_{s_{i j}}\left(v_{i j}\right)}, \overline{\lambda_{t} k_{t_{i j}}\left(v_{i j}\right)}\right)=\left(\overline{\lambda_{s}} k_{-s_{i j}}\left(v_{i j}\right), \overline{\lambda_{t}} k_{-t_{i j}}\left(v_{i j}\right)\right) .
\end{aligned}
$$

However, vectors $k_{ \pm 1}^{i}\left(v_{i j}\right)$ are linearly independent thus $\lambda_{s}=\lambda_{t}=0$.

\section{Reduced Hessian}

Let us denote the tensor of second derivatives of $\Im S_{i j}\left(g_{i j}^{0}\right)$ by $I_{i j}^{\prime}$. We are interested in the second derivatives $\Im S_{i j}^{\text {red }}\left(g_{i}, g_{j}\right)$ at $\left\{g_{k}^{0}\right\}$ (we assume $g_{5}^{0}=1$ ).

The tangent vectors to the manifold $\prod_{i=1}^{4} \mathrm{SL}(2, \mathbb{C})$ are given by

$$
V_{\text {tot }}=\{v:\{1, \ldots 5\} \rightarrow s o(1,3): v(5)=0\} .
$$

For convenience we assumed $v(5)=0$. We use here the right invariant vector fields to identify $V_{\text {tot }}$ with $T\left(\prod_{i=1}^{4} \operatorname{SL}(2, \mathbb{C})\right)$. 
Lemma 18. We have

$I_{i j}\left(v, v^{\prime}\right):=\partial^{2} \Im S_{i j}^{\mathrm{red}}\left(\left\{g_{k}^{0}\right\}\right)\left(v, v^{\prime}\right)=I_{i j}^{\prime}\left(g_{i}^{-1} \cdot v(i)-g_{i}^{-1} \cdot v(j), g_{i}^{-1} \cdot v(i)-g_{i}^{-1} \cdot v(j)\right)$,

where $I_{i j}^{\prime}(L, L)=\partial^{2} \Im S_{i j}^{\prime}(\mathbb{L}(L), \mathbb{L}(L))$.

Proof. Standard result about functions of the form $f\left(g_{j}^{-1} g_{i}\right)$. We use the right invariant vector fields, thus

$$
g_{i}=e^{-t L_{i}} g_{i}, \quad g_{j}=e^{-t L_{j}} g_{j} .
$$

We have for left invariant vector fields

$$
\partial^{2} f(L, L)=\left.\frac{d^{2}}{d t^{2}}\right|_{t=0} f\left(g e^{t L}\right) .
$$

We can now compute for $v$

$$
v(i)=L_{i}, v(j)=L_{j}, v(k)=0 \text { for } k \neq i, j .
$$

Let us compute second derivative of $F\left(g_{i}, g_{j}\right)=f\left(g_{i j}\right)$ where $g_{i j}=g_{j}^{-1} g_{i}$

$$
\partial^{2} F(v, v)=\left.\frac{d^{2}}{d t^{2}}\right|_{t=0} f\left(g_{j}^{-1} e^{t L_{j}} e^{-t L_{i}} g_{i}\right)=\left.\frac{d^{2}}{d t^{2}}\right|_{t=0} f\left(g_{j}^{-1} g_{i} e^{X(t)}\right) .
$$

We used BCH formula and commuted (we use notation $g \cdot L=g L g^{-1}$ )

$$
\begin{gathered}
e^{X(t)}=e^{t g_{i}^{-1} L_{j} g_{i}} e^{-t g_{i}^{-1} L_{i} g_{i}}, \\
X(t)=t g_{i}^{-1} \cdot L_{j}-t g_{i}^{-1} \cdot L_{i}-\frac{t^{2}}{2}\left[g_{i}^{-1} \cdot L_{j}, g_{i}^{-1} \cdot L_{i}\right]+O\left(t^{3}\right) .
\end{gathered}
$$

We use now $\partial \Im S_{i j}^{\prime}=0$ to get

$$
\partial^{2} \Im S_{i j}^{\mathrm{red}}(v, v)=\partial^{2} \Im S_{i j}^{\prime}\left(g_{i}^{-1} \cdot v(i)-g_{i}^{-1} \cdot v(j), g_{i}^{-1} \cdot v(i)-g_{i}^{-1} \cdot v(j)\right),
$$

so we found the desired result.

Lemma 19. Let us suppose that $0 \neq v \in V_{\text {tot }}$ satisfies

$$
\forall_{1 \leq i<j \leq 5} I_{i j}(v, v)=0,
$$

then there exist $1 \leq a<b \leq 4$ such that the bivectors

$$
B_{a 5}^{0}, * B_{a 5}^{0}, B_{b 5}^{0}, * B_{b 5}^{0}, B_{a b}^{0}, * B_{a b}^{0},
$$

are linearly dependent.

Proof. As all $I_{i j}$ are positive definite and $I_{i j}^{\prime}$ has the kernel spanned by

$$
B_{i j}^{0^{\prime}}, * B_{i j}^{0^{\prime}}
$$

we have

$$
I_{i j}(v, v)=0 \Leftrightarrow v(i)-v(j) \in \operatorname{span}\left\{B_{i j}^{0}, * B_{i j}^{0}\right\} .
$$

We see that from $I_{i 5}(v, v)=0$ it follows that $v(i) \in \operatorname{span}\left\{B_{i 5}^{0}, * B_{i 5}^{0}\right\}$ and thus as $v$ is nonzero there exist $i, j \neq 5$ such that

$$
0 \neq v(i) \in \operatorname{span}\left\{B_{i 5}^{0}, * B_{i 5}^{0}\right\}
$$

and also

$$
v(i)=(v(i)-v(j))+v(j) \in \operatorname{span}\left\{B_{i j}^{0}, * B_{i j}^{0}, B_{j 5}^{0}, * B_{j 5}^{0}\right\} .
$$


This means linear dependence.

Lemma 20. If the reconstructed 4-simplex (in any signature) with spacelike faces is nondegenerate then

$$
B_{a 5}^{\Delta}, * B_{a 5}^{\Delta}, B_{b 5}^{\Delta}, * B_{b 5}^{\Delta}, B_{a b}^{\Delta}, * B_{a b}^{\Delta},
$$

are linearly independent for $\{a, b, 5\}$ distinct. (see [8]).

Here by $B_{i j}^{\Delta}$ we denote geometric bivectors of the reconstructed 4-simplex

Proof. Let us assume $a=3, b=4$. The bivectors $B_{i j}^{\Delta}$ for $i, j \in\{3,4,5\}$ can be written as

$$
B_{i j}^{\Delta}=\eta_{i j} \wedge e_{12}
$$

where $\eta_{i j} \perp e_{12}$ and $e_{12}$ is the edge vector connecting vertex 1 with 2 (this edge is spacelike). Moreover, $\eta_{i j}$ are independent if the 4-simplex is nondegenerate.

Let us notice that $e_{12}\left\llcorner * B_{i j}^{\Delta}=0\right.$ and $e_{12}\left\llcorner B_{i j}^{\Delta}=-\eta_{i j}\left|e_{12}\right|^{2}\right.$.

Let us assume that there is a linear equation for the bivectors

$$
\sum_{i j \in\{3,4,5\}} \lambda_{i j} B_{i j}^{\Delta}+\lambda_{i j}^{\prime} * B_{i j}^{\Delta}=0 .
$$

Contracting it with $e_{12}$, we get

$$
\sum_{i j \in\{3,4,5\}} \lambda_{i j} \eta_{i j}=0 \Rightarrow \lambda_{i j}=0 .
$$

Taking the Hodge dual of the equation and then contracting with $e_{12}$, we get

$$
\sum_{i j \in\{3,4,5\}} \lambda_{i j}^{\prime} \eta_{i j}=0 \Rightarrow \lambda_{i j}^{\prime}=0 .
$$

Thus the bivectors are linearly independent if the reconstructed 4-simplex is nondegenerate.

Theorem 2. The reduced Hessian for the Lorentzian EPRL model (and for the Conrady-Hnybida extension for spacelike faces) is nondegenerate at the stationary point that corresponds to a nondegenerate 4-simplex.

Proof. If $H v=0$, then we are in the situation from Lemma 19. From positivity of the $I_{i j}$ it thus follows that

$$
B_{a 5}^{0}, * B_{a 5}^{0}, B_{b 5}^{0}, * B_{b 5}^{0}, B_{a b}^{0}, * B_{a b}^{0},
$$

are linearly dependent.

Let us consider now separately two cases:

1. If the stationary point corresponds to a Lorentzian 4-simplex, then

$$
B_{i j}^{0}=\tau\left(B_{i j}^{\Delta}\right)
$$

and $\tau$ preserves the space (200). By Lemma 20 we have a contradiction. 
2. If the stationary point $(+)$ corresponds to a 4-simplex solution with other signature, then there is the second point $(-)$ and

$$
B_{i j}^{+}=\tau\left(* v_{i j}^{+} \wedge N^{0}\right), \quad B_{i j}^{-}=\tau\left(* v_{i j}^{-} \wedge N^{0}\right),
$$

and $B_{i j}^{\Delta}$ has selfdual and antiself-dual parts given by $\tau^{-1}\left(B_{i j}^{ \pm}\right)$. From (200) it follows that there exist constants $\lambda_{i j}, \lambda_{i j}^{\prime}$ such that

$$
\sum_{i j \in\{a, b, 5\}, i<j} \lambda_{i j} B_{i j}^{+}+\lambda_{i j}^{\prime} * B_{i j}^{+}=0,
$$

thus taking $\overrightarrow{\mathbb{L}}_{i}^{\tau}$ and $\overrightarrow{\mathbb{K}}_{i}^{\tau}$ parts we get

$$
\sum_{i j \in\{a, b, 5\}, i<j} \lambda_{i j} v_{i j}^{+}=0, \quad \sum_{i j \in\{a, b, 5\}, i<j} \lambda_{i j}^{\prime} v_{i j}^{+}=0 .
$$

As some coefficients need to be nontrivial, we get that $v_{i j}^{+}$and thus also $B_{i j}^{+}((i, j) \in\{a, b, 5\})$ are linearly dependent. But this means that

$$
B_{a 5}^{\Delta}, * B_{a 5}^{\Delta}, B_{b 5}^{\Delta}, * B_{b 5}^{\Delta}, B_{a b}^{\Delta}, * B_{a b}^{\Delta},
$$

are linearly dependent and from Lemma 20 we have a contradiction.

Independently of the signature of the reconstructed 4-simplex the Hessian is nondegenerate.

\section{Summary}

We showed that the Hessian in the EPRL and Conrady-Hnybida (spacelike surfaces case) is nondegenerate for any stationary point (corresponding to a nondegenerate 4-simplex of either Lorentzian, Euclidean or split singnature). We also showed nondegeneracy for the Euclidean $\gamma<1$ case. Our method works fine also for $\gamma>1$, but we have not provided the details in this case. However, the method does not extend immediately to the situation when some of the faces are timelike (the asymptotic of this case was considered recently in [9]). The action in this case is purely real, and as we based our proof on the properties of imaginary part of the action, this case cannot be covered with the tools used in our paper unless they will be properly modified. The issue deserves a separate treatment and we leave this topic for future research.

\section{Acknowledgements}

We thank Marcin Kisielowski for fruitful discussions at the early stage of this project. 
Open Access. This article is distributed under the terms of the Creative Commons Attribution 4.0 International License (http://creativecommons.org/licenses/ by/4.0/), which permits unrestricted use, distribution, and reproduction in any medium, provided you give appropriate credit to the original author(s) and the source, provide a link to the Creative Commons license, and indicate if changes were made.

Publisher's Note Springer Nature remains neutral with regard to jurisdictional claims in published maps and institutional affiliations.

\section{A. Notation}

In this section we collect our notation:

1. The signature of the metric is $(+---)$.

2. Bivectors $s o(1,3)=\Lambda^{2} \mathbb{R}^{4}$ (we use identification by the scalar product). The action on vectors can be expressed as

$$
\left(v \wedge v^{\prime}\right)(w)=v\left(v^{\prime} \cdot w\right)-v^{\prime}(v \cdot w) .
$$

We define also a scalar product $(\cdot, \cdot)$ on bivectors

$$
\left(v \wedge w, v^{\prime} \wedge w^{\prime}\right)=\operatorname{det}\left(\begin{array}{ccc}
v \cdot v^{\prime} & v \cdot w^{\prime} \\
w \cdot v^{\prime} & w \cdot w^{\prime}
\end{array}\right) .
$$

Hodge star operation is denoted by $*$.

3. The adjoint action on the Lie algebra is defined by

$$
g \cdot L=g L g^{-1} \text {. }
$$

Coadjoint action on $P$ is defined by $g \cdot P(L)=P\left(g^{-1} \cdot L\right)$.

4. The Hessian is a symmetric two form (tensor) on the tangent vectors for a function $f$ at the point where the first derivative vanishes. We denote this form by $\partial^{2} f$.

5. The stationary point $\left\{g_{i}^{0},\left[\mathbb{Z}_{i j}^{0}\right]\right\}, g_{5}^{0}=1$ of the total action is referred to as the fundamental stationary point. The bivectors at this stationary point are denoted $B_{i j}^{0}$ (in the simplex frame) and $B_{i j}^{0^{\prime}}=\left(g_{i}^{0}\right)^{-1} B_{i j}^{0}$ (see the beginning of Sect. 3). The geometric bivectors $B_{i j}^{\Delta}$ are described in [8] and appear in Sect. 6.

6. Weyl spinor spaces $\mathcal{S}^{ \pm}=\mathbb{C}^{2}$ : We denote spinors from $\mathcal{S}^{+}$by $\mathbb{Z}, \mathbb{v}$, u, etc. Clifford elements for any vector $v$ are $\eta^{ \pm}(v): \mathcal{S}^{ \pm} \rightarrow \mathcal{S}^{\mp}$ fulfilling

$$
\eta^{\mp}(v) \eta^{ \pm}\left(v^{\prime}\right)+\eta^{\mp}\left(v^{\prime}\right) \eta^{ \pm}(v)=\left(v \cdot v^{\prime}\right) \mathbb{I}_{\mathcal{S}^{ \pm}} .
$$

The Lie algebra isomorphism $s o(1,3)$ to $s l(2, \mathbb{C})$ (traceless matrices) is

$$
B \rightarrow \mathbb{M}(B), \quad \mathbb{M}\left(v \wedge v^{\prime}\right)=\frac{1}{4}\left(\eta^{-}(v) \eta^{+}\left(v^{\prime}\right)-\eta^{-}\left(v^{\prime}\right) \eta^{+}(v)\right) .
$$

For two spinors $\mathbb{u}, \mathbb{v}$ we denote

$$
[\mathfrak{u}, \mathbb{v}]=\mathfrak{u}^{T} \omega \mathbb{v}, \quad \omega=\left(\begin{array}{cc}
0 & 1 \\
-1 & 0
\end{array}\right) .
$$


Every traceless matrix can be written as $\mathbb{N}=\frac{1}{2}\left(\mathfrak{u}_{+} \mathfrak{u}_{-}^{T}+\mathfrak{u}_{-} \mathfrak{u}_{+}^{T}\right) \omega$ and

$$
\mathbb{N}\left(\mathfrak{u}_{ \pm}\right)= \pm \frac{1}{2}\left[\mathfrak{u}_{-}, \mathfrak{u}_{+}\right] \mathfrak{u}_{ \pm}
$$

(see Sect. 4.2 and [8]).

7. $p^{L}$ and $p^{R}$ are left and right covectors (see Sect. 4.1) and

$$
\delta^{L} S(L)=\mathbb{L}(L) S, \quad \delta^{R} S(L)=\mathbb{R}(L) S,
$$

where $\mathbb{L}$ and $\mathbb{R}$ are left and right derivatives. We also denote $\delta=\delta^{L}$.

8. We denote

(see Sect. 4.1).

$$
\delta_{\mathbb{Z}} f(\mathbb{Z})=\left.\delta^{L} f\left(g^{-1} \mathbb{Z}\right)\right|_{g=1}
$$

9. $[\cdot]$ is a relation on spinors

$$
[\mathbb{Z}]=[\mathbb{w}] \Leftrightarrow \exists 0 \neq \lambda \in \mathbb{C}: \mathbb{Z}=\lambda \mathbb{w},
$$

thus $[\mathbb{Z}]$ is a point of $\mathbb{C P},\left[\mathbb{Z}_{i j}^{\mathbb{C}}\right]$ is a point on complexified $\mathbb{C} \mathbb{P}^{\mathbb{C}}$.

10. The vector fields of the action of $\operatorname{SL}(2, \mathbb{C})$ on $\mathbb{C P}$ are denoted by $\mathbb{L}_{\mathbb{C P}}(L)$ for $L \in s o(1,3)$. They correspond to the curves

$$
t \rightarrow\left[e^{-t L} \mathbb{Z}\right] .
$$

Similarly, the vector field of the action of $\mathrm{SL}(2, \mathbb{C})$ on $\mathcal{S}^{+}$are denoted by $\mathbb{L}_{\mathcal{S}^{+}}(L)$ for $L \in$ so $(1,3)$. They correspond to the curves

$$
t \rightarrow e^{-t L} \mathbb{Z} \text {. }
$$

11. The definition of $\overrightarrow{\mathbb{L}}_{i} \overrightarrow{\mathbb{K}}_{i}$ is in Sect. 5.2. For the twisting map $\tau$, and twisted versions $\overrightarrow{\mathbb{L}}^{\tau}, \overrightarrow{\mathbb{K}}_{i}^{\tau}$ see Sect. 5.3.

12. The vectors $k_{ \pm 1}^{i}(v)$ are defined in 5.2.

13. $X_{n, \rho}$ is a coadjoint orbit space defined in Eq. (70).

14. The projection from $X_{n, \rho}$ (coadjoint orbit) to $\mathbb{C P}$ is denoted by $\pi$. The function $f$ that is constant along the fibres can be pushed forward to $\mathbb{C P}$ and such push forward is denoted by $[f]_{\mathbb{C P}}$ (see Sect. 4.2).

15. $S^{\text {red }}, S_{i j}^{\text {red }}$ are defined in Sect. 3.1. Their Hessians are denoted by $H^{\text {red }}$ and $H_{i j}^{\mathrm{red}}$.

16. $S_{i j}^{\prime}$ and $g_{i j}=g_{j}^{-1} g_{i}$ is defined in Sect. 3.1.

17. $\mathcal{L}$ denotes Lagrangians. The subscript denotes the (part of the) action generating the given Lagrangian. We use ' to indicate Lagrangians in the coadjoint orbit space.

18. The form $I$ on the tangent space of the Lagrangian at the real point is defined in Eq. (49).

\section{References}

[1] Engle, J., Livine, E., Pereira, R., Rovelli, C.: LQG vertex with finite Immirzi parameter. Nucl. Phys. B 799, 136 (2008)

[2] Freidel, L., Krasnov, K.: A new spin foam model for 4d gravity. Class. Quantum Gravity 25, 125018 (2008)

[3] Conrady, F., Hnybida, J.: A spin foam model for general lorentzian 4-geometries. Class. Quantum Gravity 27, 185011 (2010) 
[4] Conrady, F., Freidel, L.: Semiclassical limit of 4-dimensional spin foam models. Phys. Rev. D 78, 104023 (2008)

[5] Barrett, J.W., Dowdall, R.J., Fairbairn, W.J., Gomes, H., Hellmann, F., Pereira, R.: Asymptotics of 4d spin foam models. Gen. Relativ. Gravit. 43, 2421 (2011)

[6] Barrett, J.W., Dowdall, R.J., Fairbairn, W.J., Gomes, H., Hellmann, F.: Asymptotic analysis of the EPRL four-simplex amplitude. J. Math. Phys. 50, 112504 (2009)

[7] Barrett, J.W., Dowdall, R.J., Fairbairn, W.J., Hellmann, F., Pereira, R.: Lorentzian spin foam amplitudes: graphical calculus and asymptotics. Class. Quantum Gravity 27, 165009 (2010)

[8] Kaminski, W., Kisielowski, M., Sahlmann, H.: Asymptotic analysis of the EPRL model with timelike tetrahedra. Class. Quantum Gravity 35(13), 135012 (2018)

[9] Liu, H., Han, M.: Asymptotic analysis of spin foam amplitude with timelike triangles. Phys. Rev. D 99(8), 084040 (2019)

[10] Alesci, E., Rovelli, C.: The complete LQG propagator. II. Asymptotic behavior of the vertex. Phys. Rev. D 77, 044024 (2008)

[11] Bianchi, E., Magliaro, E., Perini, C.: LQG propagator from the new spin foams. Nucl. Phys. B 822, 245-269 (2009)

[12] Bianchi, E., Ding, Y.: Lorentzian spinfoam propagator. Phys. Rev. D 86, 104040 (2012)

[13] Han, M., Zhang, M.: Asymptotics of spinfoam amplitude on simplicial manifold: Euclidean theory. Class. Quantum Gravity 29, 165004 (2011)

[14] Han, M., Zhang, M.: Asymptotics of spinfoam amplitude on simplicial manifold: Lorentzian theory. Class. Quantum Gravity 30, 165012 (2013)

[15] Barrett, J.W., Williams, R.M.: The asymptotics of an amplitude for the four simplex. Adv. Theor. Math. Phys. 3, 209-215 (1999)

[16] Barrett, J.W., Crane, L.: Relativistic spin networks and quantum gravity. J. Math. Phys. 39, 3296-3302 (1998)

[17] Alesci, E., Rovelli, C.: The complete LQG propagator: I. Difficulties with the Barrett-Crane vertex. Phys. Rev. D 76, 104012 (2007)

[18] Bonzom, V.: Spin foam models for quantum gravity from lattice path integrals. Phys. Rev. D 80, 064028 (2009)

[19] Hellmann, F., Kaminski, W.: Holonomy spin foam models: asymptotic geometry of the partition function. JHEP 10, 165 (2013)

[20] Kaminski, W., Steinhaus, S.: The Barrett-Crane model: asymptotic measure factor. Class. Quantum Gravity 31, 075014 (2013)

[21] Hörmander, L.: On the existence and the regularity of solutions of linear pseudodifferential equations. Enseign. Math. 17, 99-163 (1971)

Wojciech Kamiński

Faculty of Physics

University of Warsaw

Pasteura 5

02093 Warsaw

Poland

e-mail: wkaminsk@fuw.edu.pl 


\section{Hanno Sahlmann}

Institute for Quantum Gravity, Department of Physics

Friedrich-Alexander Universität Erlangen-Nürnberg (FAU)

Staudtstr. 7

91058 Erlangen

Germany

e-mail: hanno.sahlmann@gravity.fau.de

Communicated by Carlo Rovelli.

Received: July 29, 2019.

Accepted: August 29, 2019. 